\title{
Numerical Study of Transient Instabilities in Reverse-Roller Coating Flows
}

\author{
M.S.Chandio and M.F.Webster ${ }^{+}$ \\ Institute of non-Newtonian Fluid Mechanics, \\ Department of Computer Science, University of Wales Swansea, Singleton Park, \\ Swansea, SA2 8PP, UK.
}

\begin{abstract}
A semi-implicit Taylor-Galerkin/pressure-correction algorithm of a transient finite element form is applied to analyse the flow instabilities that commonly arise during reverse-roller coating. A mathematical model is derived to describe the solvent coating applied to the underside of the sheet, assuming that the lacquer is a Newtonian fluid and considering the flow between application roller and foil. Here, we have investigated the effects of temporal instabilities, caused by adjustment of nip-gap width and foil-position, extending our previous steady-state analysis. Foil shifting is found to have a significant influence upon pressure and lift on the foil, drag on the roller, and free coating profiles. This would result in process instabilities, such as chatter and flow-lines. In contrast, nip-gap adjustment has no influence on the coating finish.
\end{abstract}

Key words: Reverse roller-coating, application roller, sheet-foil, free-surface, numerical simulation, finite elements, transient instabilities

\section{INTRODUCTION}

This work addresses the reverse-roller coating between an application roller and a sheet-foil. The study of roller-coating with free-surfaces is an important topic that commonly arises in many industrial areas, particularly those associated with the production of tape, film and printing situations. Over the past two decades, roller-coating processes have been analysed extensively, involving experimental, analytical and computational studies. Forward roller-coating has attracted the attention of many research groups. Forward roller-coating and reverse rollercoating processes are distinguished by counter-rotating and co-rotating moving rolls, respectively. The ultimate aim of such procedures is to deposit a thin uniform layer to a continuous flexible substrate. There is little reported in the literature concerning reverse roller-coating between foil and roller, taking into account startup behaviour and time-dependent instabilities. Hence, we briefly comment upon related studies.

Cohu et al. ${ }^{4}$ conducted experimental investigations into forward roller-coating of Newtonian fluids between deformable rolls. These authors observed that the decrease of the thickness of a rubber cover on a roller, below a critical value, tends

\footnotetext{
+ Author for correspondence, Institute of Non-Newtonian Fluid Mechanics, Department of Computer Science, University of Wales Swansea, Singleton Park, Swansea SA2 8PP, UK,

Email: $\underline{\text { M.F.Webster@swan.ac.uk }}$
} 
to decrease the coating thickness significantly. Based on forward roller-coating Carvalho et al. ${ }^{5}$ have argued in their numerical work, that the upstream free-surface touches the top roll, and air is trapped between the roll surface and the coating liquid. Consequently, the coated film that is delivered is defective. Various flow states are described, both metered and premetered, by moving the rolls apart and bringing them together. As the rolls are pushed together, the gyre moves upstream towards the inlet plane. These authors have replaced the fixed inlet film thickness condition with a weighted kinematic residual, which guarantees that the flow is normal to the inlet boundary in some averaged sense.

Marcio et al. $^{6}$ conducted a similar study, where the stability of the given system to transverse perturbation is analysed theoretically, numerically and experimentally. A mathematical model is presented to predict the critical capillary number for the onset of ribbing, concluding that roll cover deformation alters the wavelength of the ribbing pattern. Roll cover softness is related to increasing the solids elasticity number. Increasing the elasticity number of the soft roll cover, largens the wavelengths and diminishes wave numbers, and this stimulates the faster increasing instability modes. The consequence is a larger ribbing wavelength and the retardation of the levelling rate. To obtain a desired coating thickness, an appropriate criterion must be selected to pinpoint the choice of roll covers.

Fourcade et al. ${ }^{7}$ investigated a coating operation of a reverse roller-coating process between two rollers. The main attention is focused on the deformation of the elastomer on the coated roll. The pressure is reported to increase in the converging section of the gap, and reaches its peak slightly to the left of the contact point of the rollers. The largest deformation of the elastomer cover of $70 \mu \mathrm{m}$ is observed to occur at the location where the pressure reaches a maximum. The lower the gap size, the higher the pressure peak that is observed. Chen and Scriven ${ }^{8}$ chose to set the inflow rate based upon the incoming liquid layer, treated as a plug flow following Benjamin ${ }^{9}$. According to Benjamin, in meniscus coatings, the flow is always pre-metered and, therefore, imposing a velocity profile at the inflow boundary is a satisfactory boundary condition.

One difficulty with computer modelling of such coating scenarios lies in the treatment of moving free-surface problems, accommodating kinematic and dynamic boundary conditions on the free-surface and the simultaneous calculation of its position. For the last two decades the finite element method has played an important role in simulating the flow of fluids subject to free surfaces. Literature of relevance on this topic can be found in ${ }^{10-19}$. For details on this issue we refer to our previous steady analysis ${ }^{18}$, where attention is focused upon the flow patterns that result and variation in pressure, lift and drag at various roll and foil-speeds. Here, a finite element simulation of the roller-coating process is presented, based on a semi-implicit Taylor-Galerkin/Pressure-correction algorithm ${ }^{1-3}$. For free-surface prediction, we use kinematic boundary adjustment with a mesh-stretching algorithm.

The main purpose of the present work is to provide a description of reverse roller-coating flows, accounting for the parameters affecting the coating process, and, in particular, those of most practical significance. Major attention is focused upon flow instabilities, seeking to investigate transient effects and the influence 
these have upon typical processing instabilities. Various nip-flow conditions are considered, to take into account some leakage at the nip and to provide consistent local settings. The effects of such leakage upon the flow are determined. In particular, temporal variations between leakage and no-leakage states are simulated. Finally, temporal foil-shifting is investigated. Foil-shifting is invoked in two distinct forms: first, via global uniform shifting, and second, through more local adjustment. Results are interpreted through pressure, lift and drag, quantified locally, and through the influence of such temporal fluctuations upon the freesurface shape of the lacquer coating.

\section{PROBLEM SPECIFICATION}

The isothermal and two-dimensional coating flow of Newtonian liquid is considered. The system consists of a roller of radius $r_{1}$, rotating at speed $\omega(t)$, applying a coating to the underside of the alloy sheet of thickness $G(\mathbf{x}, t)$. The sheet rests on both the roller and the thin film of fluid trapped between the roller and the sheet. The sheet moves with speed $\mathrm{U}_{\text {foil }}$ in the horizontal direction (negative $\mathrm{x}$ ) and the problem is posed in a Cartesian frame of reference. A schematic diagram of the flow is illustrated in Fig. 1.

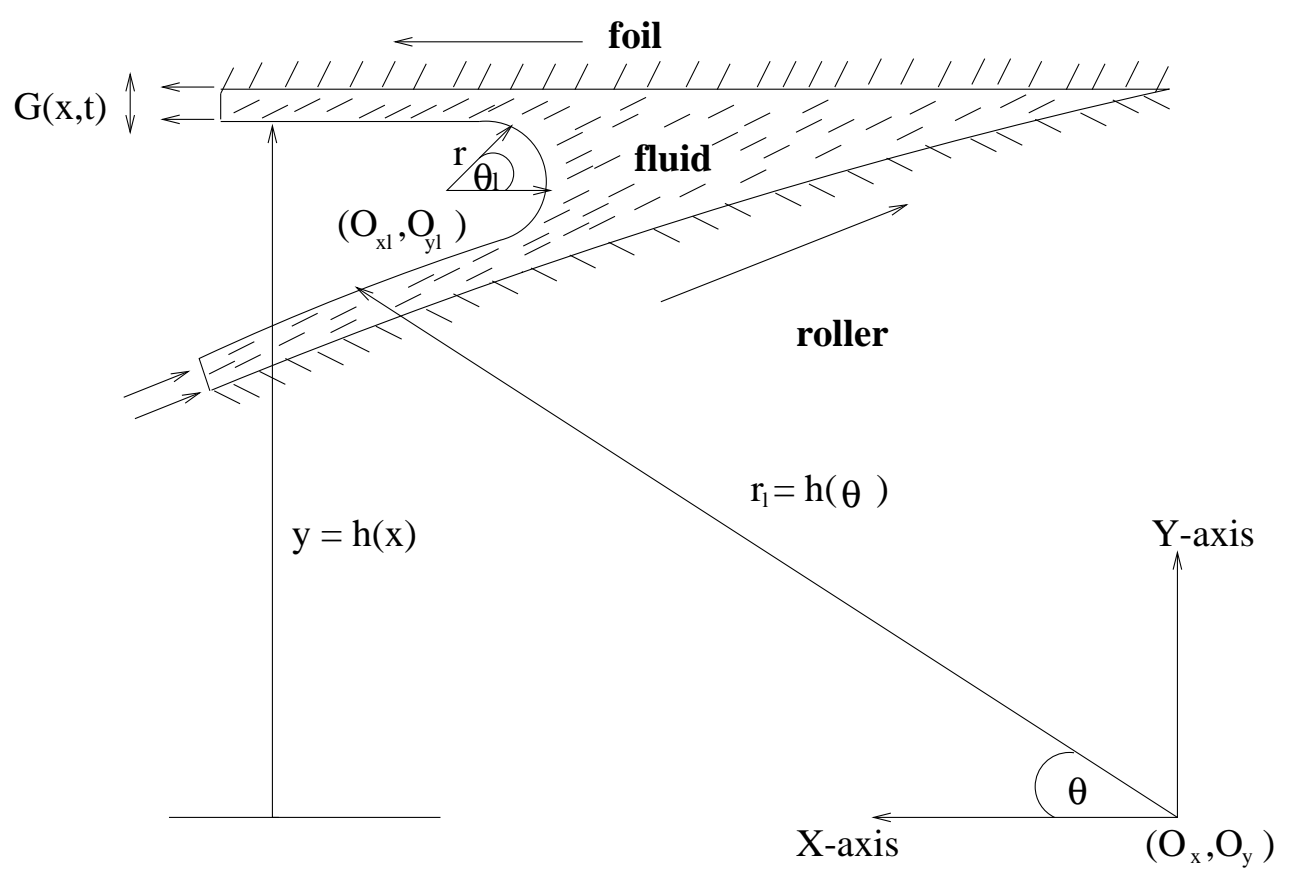

Figure 1. Schematic flow diagram 
(a) full mesh

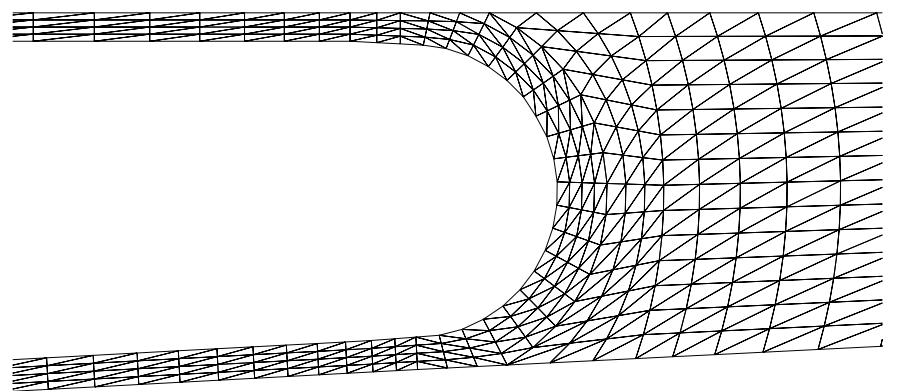

(b) free-surface mesh

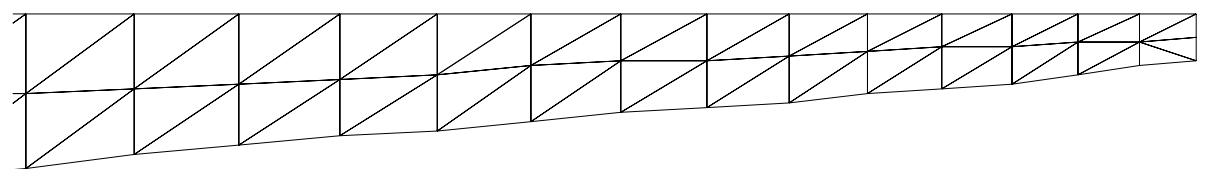

(c) mesh at nip

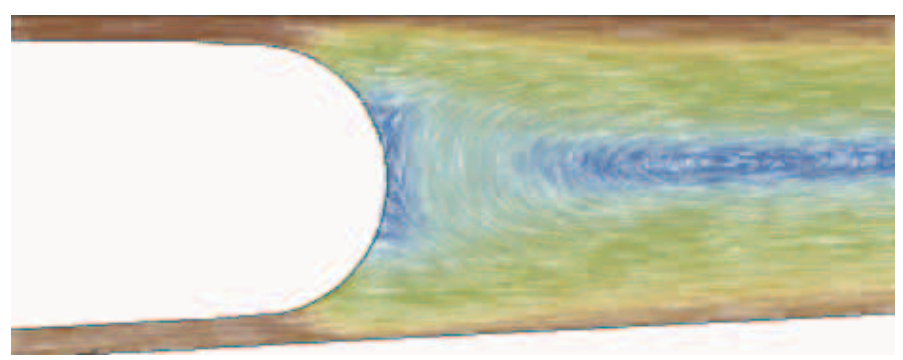

(d) flow via motion blur

Figure 2: Finite element mesh sections and flow representation

\section{GOVERNING EQUATIONS}

The isothermal flow of Newtonian fluid is governed by equations for the conservation of mass and transport of momentum. In the absence of body forces, the system may be expressed in the form

$$
\begin{aligned}
& \rho \frac{\partial \mathrm{u}}{\partial t}=\nabla . \tau-\rho \mathrm{u} . \nabla \mathrm{u}-\nabla p \\
& \nabla . \mathrm{u}=0
\end{aligned}
$$

where $\rho$ is the fluid density, $t$ is the time, $u(x, t)$ is the fluid velocity and $p$ is the isotropic pressure. For Newtonian flows, the stress $\tau$ is defined via a constant viscosity $\mu$, and the rate of deformation tensor $\mathbf{D}$, 


$$
\begin{aligned}
& \tau=2 \mu \mathbf{D} \\
& \mathbf{D}=\frac{\mathrm{L}+\mathrm{L}^{\mathrm{t}}}{2} \text { and } \mathrm{L}=\nabla \mathrm{u} .
\end{aligned}
$$

For a Newtonian fluid, the Navier-Stokes equations can be recovered, by recourse to the continuity equation (2),

$$
\rho \frac{\partial \mathrm{u}}{\partial t}=\mu \nabla^{2} \mathrm{u}-\rho \mathrm{u} . \nabla \mathrm{u}-\nabla p
$$

where $\mu \nabla^{2} \mathrm{u}$ is a diffusion term.

For conciseness and convenience, adopting characteristic scales on velocity, $\mathrm{U}$, length, $\mathrm{L}$ and viscosity, $\mu$, we may define non-dimensional variables $\mathrm{u}=\mathrm{Uu}^{*}$ and $\mathrm{p}=[\mu \mathrm{U} / \mathrm{L}] \mathrm{p}^{*}$. Hence, we may define an equivalent non-dimensional system of equations to (2) and (5), discarding the * notation for clarity of representation,

$$
\begin{aligned}
& \operatorname{Re} \frac{\partial \mathrm{u}}{\partial t}=\nabla^{2} \mathrm{u}-\operatorname{Reu} . \nabla \mathrm{u}-\nabla p, \\
& \nabla . \mathrm{u}=0,
\end{aligned}
$$

where the non-dimensional group Reynolds number is defined as $\operatorname{Re}=\frac{\rho U L}{\mu}$.

For the solution of the given system of governing equations, both initial and boundary conditions are required. Initial conditions can be formed by prescribing initial values for the primitive field variables at $t=0$,

$$
\begin{aligned}
& \mathrm{U}(\mathrm{x}, \mathrm{t})=\mathrm{u}_{0}(\mathrm{x}, 0), \\
& \mathrm{p}(\mathrm{x}, \mathrm{t})=\mathrm{p}(\mathrm{x}, 0) .
\end{aligned}
$$

Conditions at the free-surface require a normal constraint,

$$
\mathrm{p}+\tau_{\mathrm{nn}}=-\mathrm{p}_{0}+\sigma_{\mathrm{s}} \beta
$$

whilst the absence of friction ensures the tangential constraint,

$$
\tau_{\text {nt }}=0
$$

Here, $\tau_{\mathbf{n n}}, \tau_{\mathbf{n t}}$ are normal and tangential stress components, respectively, $\mathrm{p}_{0}$ is atmospheric pressure and $\mathrm{p}$ local pressure, $\sigma_{\mathrm{s}}$ is a surface tension coefficient and $\beta$ is the mean curvature of the free-surface ${ }^{14}$. Remaining boundary conditions are taken of no-slip on roller and foil, uniform flow at inlet on the roller and outlet on the foil. In a no-leakage state, there is vanishing flux across the nip. No-slip boundary conditions for the flow on solid surfaces are taken as:

$$
\begin{aligned}
& \text { on foil: } U_{x}=-U_{\text {foil }}, U_{y}=0 ; \\
& \text { on roller: } U_{x}=U_{\text {roll }} \cos \theta, U_{y}=U_{\text {roll }} \sin \theta \text {; }
\end{aligned}
$$

where $\mathrm{U}_{\text {roll }}=\mathrm{R} \omega, \mathrm{R}$ is the radius and $\omega$ the angular rotation rate of the roller. 
The evolving position of the free-surface, is unknown apriori and must therefore be computed as part of the scheme. In this respect, we appeal to the kinematic boundary conditions ${ }^{10}$. On flat free-surface boundaries (lines at constant y) this leads to:

$$
\frac{\partial h}{\partial t}=-U_{x} \frac{\partial h}{\partial x}+U_{y}
$$

On curved meniscus boundary sections (lines at fixed azimuthal angle $\theta$ setting), we have:

$$
\frac{\partial h}{\partial t}=-U_{\vartheta} \frac{1}{r} \frac{\partial h}{\partial \vartheta}+U_{r}
$$

A combination of both forms (10) and (11) is required for the current flow problems.

\section{FINITE ELEMENT ANALYSIS}

A Taylor-Galerkin algorithm is used to solve the governing equations (6). This involves a two-step Lax-Wendroff approach, based on a Taylor series expansion up to second order in time, to compute solutions through a time stepping procedure. A two-step pressure-correction method is applied to handle the incompressibility constraint. Employing a Crank-Nicolson treatment on diffusion terms, produces an equation system of three fractional-staged equations ${ }^{2}$. In stage one a non-solenodal velocity field $\mathrm{u}^{\mathrm{n}+1 / 2}$ and $\mathrm{u}^{*}$ are computed via a predictorcorrector doublet. The resulting mass-matrix bound equation is solved via a Jacobi iteration. With the use of $\mathrm{u}^{*}$, the second stage computes the pressure difference, $\mathrm{p}^{\mathrm{n}+1}-\mathrm{p}^{\mathrm{n}}$, via a Poisson equation, and the application of a direct Choleski solver. The third stage completes the time step loop, calculating the end-of-time-step solenoidal velocity field $\mathrm{u}^{\mathrm{n}+1}$, again by a Jacobi iterative solver. The details upon this implementation may be found in Townsend et al. ${ }^{1,2}$.

Following the notation of Cuvelier et al. ${ }^{20}$, the velocity and pressure fields are approximated by $\mathrm{U}(\mathbf{x}, \mathrm{t})=\mathrm{U}^{\mathrm{j}}(\mathrm{t}) \phi_{\mathrm{j}}(\mathbf{x})$ and $\mathrm{P}(\mathbf{x}, \mathrm{t})=\mathrm{P}^{\mathrm{k}}(\mathrm{t}) \psi_{\mathrm{k}}(\mathbf{x})$, where $\mathrm{U}$ and $\mathrm{P}$ represents the vectors of nodal values of velocity and pressure, respectively, and $\phi_{\mathrm{j}}$ are piecewise quadratic and $\psi_{\mathrm{k}}$ linear basis functions.

The fully-discrete semi-implicit Taylor-Galerkin/pressure-correction system of equations may be expressed in matrix form as,

$$
\begin{array}{ll}
\text { Stage 1a } & \left(\frac{2 \operatorname{Re}}{\Delta t} M+\frac{1}{2} S\right)\left(U^{n+\frac{1}{2}}+U^{n}\right)=\left\{-[S+\operatorname{Re} N(U)] U+L^{T} P\right\}^{n} \\
\text { Stage 1b } & \left(\frac{\operatorname{Re}}{\Delta t} M+\frac{1}{2} S\right)\left(U^{*}-U^{n}\right)=\left(-\left[S U+L^{T} P\right]^{n}-[\operatorname{Re} N(U) U]^{n+\frac{1}{2}}\right.
\end{array}
$$




$$
\begin{array}{ll}
\text { Stage } 2 & K\left(P^{n+1}-P^{n}\right)=-\frac{2}{\Delta t} L U^{*} \\
\text { Stage } 3 & \frac{1}{\Delta t} M\left(U^{n+1}-U^{*}\right)=\frac{1}{2} L^{T}\left(P^{n+1}-P^{n}\right),
\end{array}
$$

where $M, S, N(U), L$, and $K$ are consistent mass matrix, momentum diffusion matrix, convection matrix, pressure gradient matrix and pressure stiffness matrix, respectively. With elemental fluid area $\mathrm{d} \Omega$, such matrix notation implies,

$$
\begin{aligned}
& M_{i j}=\int_{\Omega} \phi_{i} \phi_{j} d \Omega \\
& N(U)_{i j}=\int_{\Omega} \phi_{i}\left(\phi_{l} U_{l} \frac{\partial \phi_{j}}{\partial x}+\phi_{l} U_{l} \frac{\partial \phi_{j}}{\partial y}\right) d \Omega \\
& \left(\left(L_{k}\right)_{i j}\right)=\int_{\Omega} \frac{\partial \phi_{j}}{\partial x_{k}} d \Omega \\
& K_{i j}=\int_{\Omega} \nabla \psi_{i} \nabla \psi_{j} d \Omega \\
& S_{i j}=\int_{\Omega} \nabla \phi_{i} \nabla \phi_{j} d \Omega .
\end{aligned}
$$

For the computation of free-surface, we have used the kinematic boundary conditions, (10-11). These can be expressed in a general discrete variational form,

$$
\begin{aligned}
& \frac{1}{\Delta t} \int_{\Gamma_{F}}\left(\phi_{i}+\left(\begin{array}{ll}
\alpha_{1} & u . \nabla
\end{array}\right) \phi_{i}\right)\left(\psi_{k}+\left(\begin{array}{ll}
\alpha_{2} & u . \nabla
\end{array}\right) \psi_{k}\right) \Delta H_{k}^{n+1} d \Gamma_{F}= \\
& -\int_{\Gamma_{F}}\left(\phi_{i}+\alpha_{1} \quad u . \nabla \phi_{i}\right)\left(u . \nabla \psi_{k}\right) H_{k}^{n} d \Gamma_{F}
\end{aligned}
$$

adopting notation for time-step, $\Delta \mathrm{t}$, interpolant, $H^{\mathrm{n}}(\mathrm{x})$, interpolating functions, $\psi_{\mathrm{k}}(\mathrm{x})$, and nodal solution increment, $\Delta H_{k}^{n}$,

$$
H^{n}(x)=H_{k}^{n} \psi_{k}(x) \text { and } \Delta H_{k}^{n+1}=\left(H_{k}^{n+1}-H_{k}^{n}\right) .
$$

The scheme expressed in (13) is quite flexible, where we define generalized scalar factors $\alpha_{i}$ to switch between Galerkin and Streamline Upwind Petrov Galerkin (SUPG) (explicit and implicit) schemes, as and when required. A freesurface boundary segment is indicated by $\Gamma_{\mathrm{F}}$, over which quadrature may be established. In equation (13), the generalized form of convective term is represented, subsuming either equation (10) or (11), depending upon the particular boundary segment under consideration. We have found it most effective to use $\alpha_{1}=$ $\alpha^{\mathrm{h}}$ (an SUPG parameter ${ }^{3}$ ) and $\alpha_{2}=\Delta \mathrm{t} / 2$ to recover an implicit SUPG scheme. Then, 
both $\phi_{i}$ and $\psi_{k}$ are taken as linear functions on straight-sided boundary element sections.

\section{$5 \quad$ NUMERICAL RESULTS AND DISCUSSION}

A standard foil-speed setting of one unit and roller speed, $90 \%$ of foil-speed, constitutes the base scenario around which solutions are sought. Results are reported in non-dimensional form for convenience of representation. The mesh used is displayed in fig. 2, with 2925 nodes, 1302 elements and 6662 degrees of freedom. A typical steady-state flow pattern is shown in fig. $2 \mathrm{~d}$, represented in space-filled motion blur format, based on the velocity vector field ${ }^{18}$. In this section, we are particularly interested in pressure maxima across the nip region (see fig. 2), lift is considered along the foil surface and drag on the roller. For a Newtonian fluid, the lift, drag and stress are given through the following expressions:

$$
\begin{aligned}
& L_{\text {foil }}=\int_{\Gamma_{\text {foil }}}\left\{-p+\tau_{y y}\right\} d \Gamma, \\
& D_{\text {roller }}=\int_{\Gamma_{\text {roller }}}\left\{-p \cos \vartheta+\tau_{x x} \cos \vartheta+\tau_{x y} \sin \vartheta\right\} d \vartheta .
\end{aligned}
$$

Results are categorised into different sections. The first deals with the variation in nip velocity and pressure profile conditions. This is in order to choose a suitable velocity profile at the nip, prior to investigating temporal changes. A second section is devoted to the study of temporal changes in leakage, but without foil-shifting. This allows us to interpret transient variations of pressure at the nip. Global foilshifting at steady-state is studied in a third section. Lastly, we consider foil-shifting locally and globally in time. All settings discussed are concerned with nodal positions $\mathrm{N}_{2}, \mathrm{~N}_{4}$ and $\mathrm{N}_{5}$. No-slip boundary conditions (9) apply on nodes $\mathrm{N}_{1}$ and $\mathrm{N}_{3}$, see fig. 3. In the standard no-leakage setting, fluid is not permitted to traverse through the nip-gap. So fluid travels along the roller and is carried away by the foil. The parameter of importance here in the numerical algorithm, is the time-step $(\Delta t)$. This is chosen for pragmatic reasons, to satisfy accuracy and stability constraints, as 0.005 units.

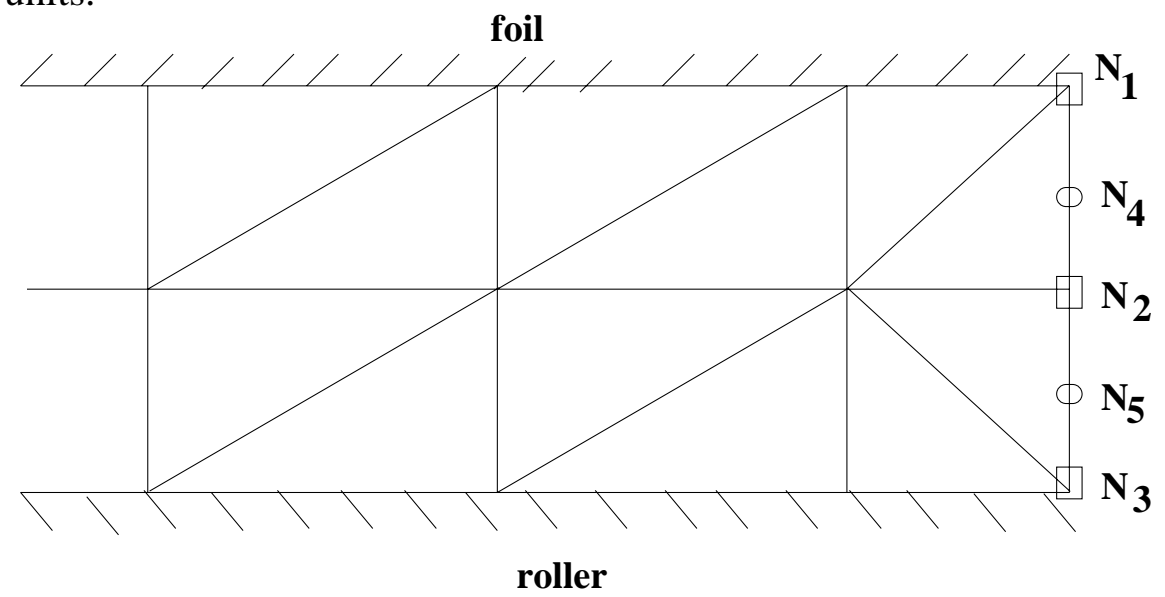

(a) Nip mesh 


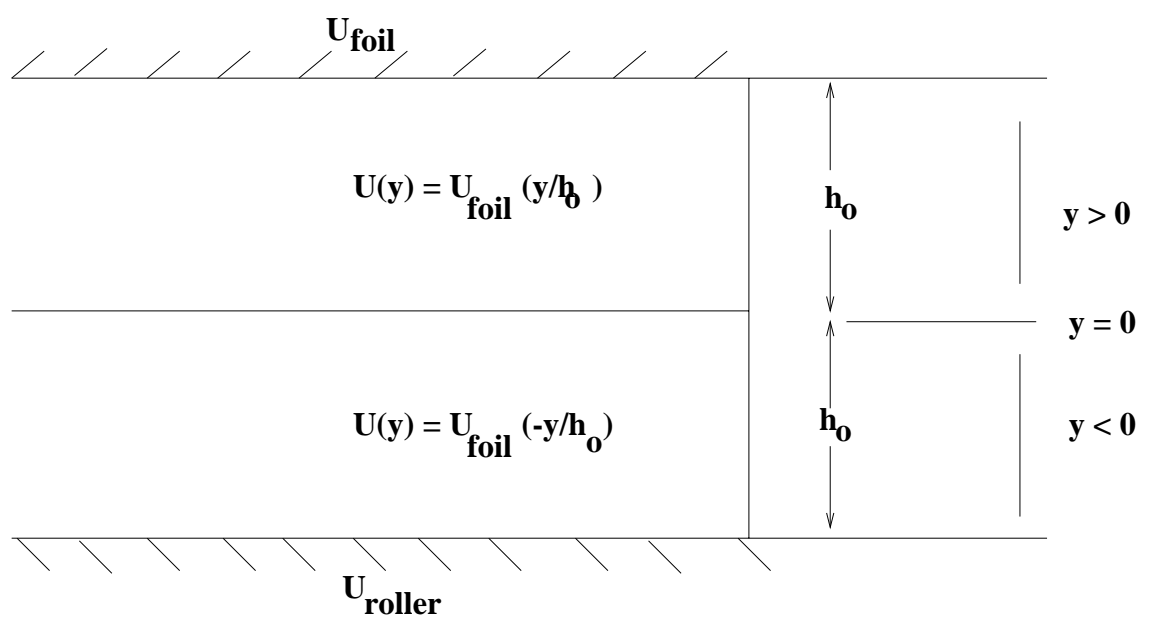

(b) Nip velocity conditions

Figure 3. Nip mesh and velocity conditions

\subsection{Variation in nip flow conditions}

Prior to analysing flow instabilities, it is instructive to choose an appropriate velocity profile to allow a degree of leakage at the nip. Temporal nip boundary conditions are consigned to later sections. There is no flow through the nip under standard settings. No-slip boundary conditions apply on the foil and roller node $\left(\mathrm{N}_{3}\right)$ at the nip, see fig.3. In fig. 5, various velocity (and pressure) profiles of plug and Couette-type are specified at a fixed nip-gap width. At nodes $\mathrm{N}_{2}, \mathrm{~N}_{4}, \mathrm{~N}_{5}$ corresponding values are charted in Tables 1 and 2. A typical schematic diagram of plug and Couette flow is shown in fig. 4. Natural conditions are referred to as free, strong conditions as fixed. In setting I, velocity and pressure are free at all three nodes. In setting II, velocity is set free and pressure vanishes at node $\mathrm{N}_{2}$. The resulting velocity profile of settings II is imposed in setting III, whilst pressure remains unspecified. Split plug flow profiles are imposed in setting IV. Lastly, a Couette flow profile, with $\mathrm{U}$ free, but vanishing at $\mathrm{N}_{2}$ is specified in settings $\mathrm{V}$ and VI, respectively.

It is observed that pressure, lift and drag attain the most elevated values at the unconstrained velocity and pressure profiles at the nip. This is due to high local nip pressures that raise the flow rate at the nip section. In order to retain stability and to avoid numerical divergence, it is imperative to fix either $\mathrm{u}$ or $p$ at the nip. Imposing either a plug or Couette flow velocity profile brings the solution close to the standard setting, with a Couette flow profile being the more consistent with the flow, see fig. $5 \mathrm{~d}$ and Tables 1 and 2 . 

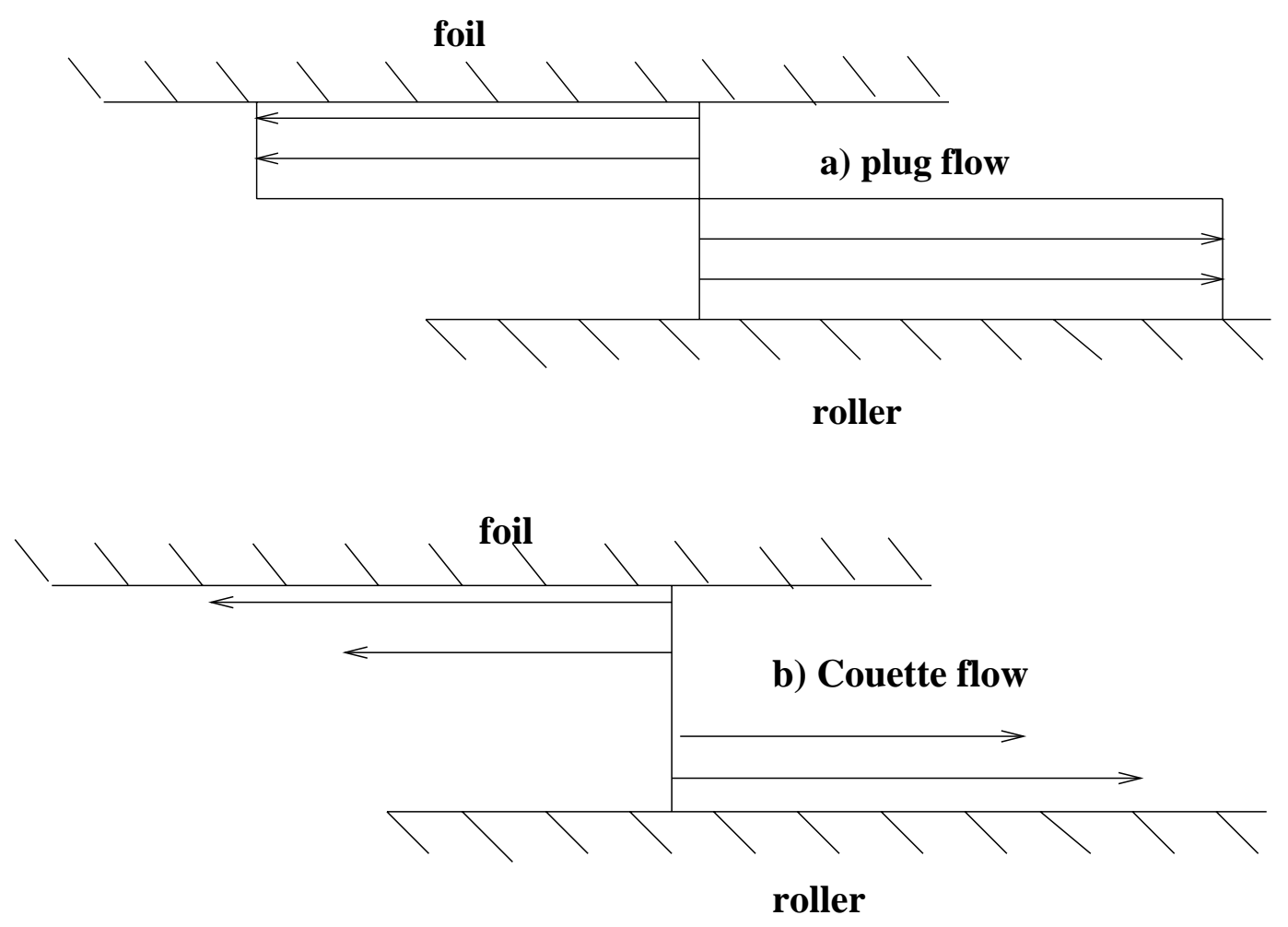

Figure 4. Schematic diagram of a) Plug and b) Couette flow profiles at nip.

It is noted in fig.6, that the maximum lift around $82 * 10^{3}$ units, is observed on the nip outlet with the Couette flow setting, see fig.5d. With the standard noleakage setting, a negligible leakage is observed through the nip-gap, inherent to the FE discretisation, and the lift value is $63^{*} 10^{3}$ units. With a Couette flow setting, a larger degree of leakage is allowed that generates larger lift than the standard setting. Differences in total lift across the various settings are practically identical, as shown in Table 2. As pressure decreases, lift and drag also decrease in equal proportion, see Table 2 . Since these changes are purely local and are restricted to the nip region, so the free-surface remains unaffected.

\begin{tabular}{|c|c|c|c|c|c|c|}
\hline \multirow{2}{*}{ settings } & \multicolumn{4}{|c|}{$\mathrm{U}$} & \multicolumn{3}{c|}{$\mathrm{V}$} \\
\cline { 2 - 7 } & $\mathrm{N}_{2}$ & $\mathrm{~N}_{4}$ & $\mathrm{~N}_{5}$ & $\mathrm{~N}_{2}$ & $\mathrm{~N}_{4}$ & $\mathrm{~N}_{5}$ \\
\hline standard & 0 & 0 & 0 & 0 & 0 & 0 \\
\hline I & -399 & 384 & 383 & - & 0.009 & - \\
\hline II & -0.933 & -1.2 & -0.24 & -0.06 & -0.36 & -0.46 \\
\hline III & $=$ & $=$ & $=$ & $=$ & $=$ & $=$ \\
\hline IV & 0 & $\mathrm{~N}_{1}$ & $\mathrm{~N}_{3}$ & 0 & $\mathrm{~N}_{1}$ & $\mathrm{~N}_{3}$ \\
\hline V & -1.8 & $\mathrm{U}_{\text {foil }}\left(\mathrm{h}_{0-\mathrm{y}}\right)$ & $\mathrm{U}_{\text {roller }}\left(\mathrm{h}_{0}+\mathrm{y}\right)$ & 0 & 0 & 0 \\
\hline VI & 0 & $\mathrm{U}_{\text {foil }}\left(\mathrm{h}_{0}-\mathrm{y}\right)$ & $\mathrm{U}_{\text {roller }}\left(\mathrm{h}_{0}+\mathrm{y}\right)$ & 0 & 0 & 0 \\
\hline
\end{tabular}

Table 1. Nip conditions; velocity 


\begin{tabular}{|c|c|c|c|c|}
\hline \multirow{2}{*}{ settings } & \multicolumn{2}{|c|}{$\mathrm{P}$} & \multirow{2}{*}{ Lift } & \multirow{2}{*}{ Drag } \\
\cline { 2 - 3 } & Nip $_{\text {outlet }}$ & Nip $_{\text {foil }}$ & & \\
\hline standard & 160 & 160 & 1311 & 1.45 \\
\hline I & 614 & $633-655$ & 100639 & 2965 \\
\hline II & $0.642-0.650$ & $26-61$ & 776 & -0.47 \\
\hline III & 20.03 & $41.2-68.3$ & 839 & -0.25 \\
\hline IV & 152 & $150-121$ & 1286 & 1.45 \\
\hline V & 85 & $95-102$ & 1286 & 1.45 \\
\hline VI & 156 & $153-123$ & 1298 & 1.45 \\
\hline
\end{tabular}

Table 2. Nip conditions; $\mathrm{P}, \mathrm{L}_{\mathrm{f}}$ and $\mathrm{D}_{\mathrm{R}} * 10^{3}$ units

\subsection{Temporal adjustment of nip conditions}

The main purpose here is to evaluate the effect of temporal leakage adjustment upon the pressure and lift acting on the foil, with a fixed nip-gap width. This is performed through variations in conditions across the time steps with a corresponding sensitivity analysis. The pressure variation is observed at various time step setting protocols, by switching between standard setting (no leakage) and Couette flow setting (leakage setting VI, see Table 1) in time. Pressure, lift and drag reflect almost identical results to those at standard settings, see fig.7 and Table 3. With temporal adjustment of nip conditions, but without foil movement, we observe in fig.8, a trend towards a constant periodicity (regular frequency) in the temporal change of the pressure at the nip. There is only minimal pressure variation and this is local to the nip. Hence, there is hardly any influence over the coating free-surface profile on the foil.

\begin{tabular}{|l|c|c|cc|cr|}
\hline protocol & $\mathrm{P}^{\text {mean }}$ & ampl. & \multicolumn{2}{|c|}{ Lift } & \multicolumn{2}{c|}{ Drag } \\
& & & $\max$. & $\min$ & $\max$ & $\min$ \\
\hline alternate $\Delta \mathrm{t}$ & 157.9 & 0.05 & 1296 & 1293 & 1.255 & 1.242 \\
\hline $10_{\mathrm{l}}-10_{\mathrm{nl}}$ & 156.8 & 0.25 & 1296 & 1293 & 1.251 & 1.246 \\
\hline $20_{\mathrm{l}}-50_{\mathrm{nl}}$ & 157.3 & 0.55 & 1299 & 1293 & 1.253 & 1.246 \\
\hline $50_{\mathrm{l}}-50_{\mathrm{nl}}$ & 157.8 & 0.75 & 1309 & 1300 & 1.378 & 1.370 \\
\hline $100_{\mathrm{l}}-100_{\mathrm{nl}}$ & 157.8 & 1.25 & 1309 & 1300 & 1.378 & 1.370 \\
\hline std. setting & 159.8 & - & \multicolumn{2}{|c|}{1311} & \multicolumn{2}{c|}{1.45} \\
\hline
\end{tabular}

Table 3. Temporal leakage adjustment; $\mathrm{P}^{\text {mean }}, \mathrm{L}_{\mathrm{f}}$ and $\mathrm{D}_{\mathrm{R}} * 10^{3}$ units

\subsection{Global foil-shifting}

Thus far, we have observed that there is only minimal pressure variation and this is local to the nip. Hence, foil/meniscus pressure profiles remain unaffected. Next, the effects of foil-shifting are investigated in response to flow-instabilities. Nip-gap size is increased by shifting the foil vertically upwards, uniformly across its length, relocating at a specified new nip-width, taken as a function of time-step variation. 


\subsubsection{Shift and solve to steady state}

The variation of leakage is considered at various nip-width settings. At $2 \%$ nip-width, the pressure peak at the nip has decreased by $83 \%$ to that of the standard setting result. Correspondingly, there is a decline of similar form in lift and drag, see Table 4 . Locally to the nip, lift decreases by $68 \%$ and drag by $60 \%$. This is shown to be largely restricted to the nip region, see fig.9. Similarly, at $3 \%$ nipwidth, pressure maxima at the nip has decreased by $90 \%$, whilst lift and drag are also decreased by $78 \%$ and $88 \%$, respectively, see Table 4 .

\begin{tabular}{|c|c|c|c|c|}
\hline $\begin{array}{c}\text { Nip-width(units) } \\
\% \text { of coat-outlet }\end{array}$ & Leakage(units) & $\mathrm{P}_{\text {nip }}$ & Lift & Drag \\
\hline $1 \%, 0.01$ & nil & 160 & 1311 & 1.45 \\
\hline $2 \%, 0.02$ & 0.0044 & 27 & 424 & 0.578 \\
\hline $3 \%, 0.03$ & 0.0067 & 16 & 289 & 0.185 \\
\hline
\end{tabular}

Table 4. Global foil shifting to steady state; $\mathrm{P}_{\text {nip }}, \mathrm{L}_{\mathrm{f}}$ and $\mathrm{D}_{\mathrm{R}} * 10^{3}$ units

Such global foil shifting, considered to steady state in time, hardly affects the outlet/meniscus flow. This is entirely reasonable as the adjustment incurs such minor leakage, which hardly reduces the flow rate at the outlet, i.e. coating on the foil. We may discern the influence of nip-width adjustment, prior to transient fluctuations. That is, in contrast to the Couette flow study of section 5.2, where an increasing degree of leakage also applied, but imposed for a $1 \%$ fixed nip-gap width. The findings are broadly similar with localized force balance adjustments restricted to the nip, so that the free-surface remains unaffected. Hence, an important point to note is that changes imposed in nip-gap width have influence around the nip region only.

\subsubsection{Temporal adjustment of foil position}

Foil vibration is performed at set time-steps intervals. So, for example, the foil is allowed to move up/down by creating/removing a gap between roller and foil, after every $\mathrm{N}$ alternate time-steps. Effectively the foil is either rising off/laying on the roller. Nip conditions, that switch between different foil positions, are taken as of sub-section 5.2. The results presented below are associated with data sampled when the foil is in a shift-up mode, and are compared with the standard setting. The foil is shifted at different time-step protocols. The drop of pressure is not related only to nip-width, but also to foil shift-up time. Increment in foil shift-up time retards the pressure and lift considerably, see Table 5. It is found helpful to consider integrated quantities on surfaces in their contributions per unit area (distributional), to appreciate their spread. As regards distributional pressure, lift and drag on the foil in a no-leakage (nl) state, it is noted that, the greater time-step variation protocol $\left(100_{1}-100_{\mathrm{nl}}\right)$ does attain a level, close (within 10\%) to that of the standard nl-setting, see figs.10-11. Pressure profiles, are illustrated in contrast to the standard setting. The decline and rise of pressure is clearly exhibited, at leakage/no-leakage 
states, across time-step variations, see fig. 11. On average, pressure, lift and drag decrease, with increasing nip-width, see Table 5.

Temporal variation in pressure is sampled at a single point on the foil at the nip region, see fig.12. Pressure is observed to be a direct sensor of lift, and hence, the choice to plot this quantity. The rise and fall of pressure is clearly apparent at alternate specified time-steps. Therein, we see regular periodicity in pressure sampled at the nip. The sharpness of the profiles, over an individual period is associated with high frequency protocols. At low frequency, this sharpness is dispersed, such as with the $100_{1}-100_{\text {nl }}$ protocol. This is so, even with increase in nip-gap width cases.

\begin{tabular}{|l|c|c|c|c|}
\hline Force & Nip-width & $10_{\mathrm{up}}-10_{\text {reset }}$ & $20_{\mathrm{up}}-50_{\text {reset }}$ & $100_{\mathrm{up}}-100_{\text {reset }}$ \\
\hline Pressure & $2 \%$ & $31.5-57.6$ & $37.8-102$ & $11.5-113$ \\
\cline { 2 - 5 } & $3 \%$ & $15.6-46.8$ & $17.4-94.6$ & $1.9-113$ \\
\cline { 2 - 5 } & $5 \%$ & $2.2-38.6$ & $8.5-88.8$ & $-3.2-113$ \\
\hline \multirow{4}{*}{ Lift } & $2 \%$ & $407.5-805$ & $408.3-1128$ & $277-1186$ \\
\cline { 2 - 5 } & $3 \%$ & $228.2-722$ & $216.4-1096$ & $136-1181$ \\
\cline { 2 - 5 } & $5 \%$ & $49.4-649$ & $37.5-1071$ & $13.1-1181$ \\
\hline \multirow{4}{*}{ Drag } & $2 \%$ & $0.475-1.0$ & $0.555-1.32$ & $0.396-1.22$ \\
\cline { 2 - 5 } & $3 \%$ & $0.278-0.95$ & $0.35-1.31$ & $0.22-1.22$ \\
\cline { 2 - 5 } & $5 \%$ & $0.062-0.9$ & $0.136-1.31$ & $0.22-1.22$ \\
\hline
\end{tabular}

Table 5. Foil shifting (globally); temporal variations $* 10^{3}$ units

We comment that by employing an appropriate nip-width setting, one can control the threshold level of pressure. This may be used as a mechanism to constrain lift, which mitigates foil-vibration. The plots of Fig. 13 for $\mathrm{P}_{\min }(\mathrm{t})$ in a leakage-state, for 2\%, 3\% and 5\% settings, at high and low frequency protocols, indicate corresponding settling times (to a steady-position). These are more rapid at larger nip-widths. Permitting a leakage/no-leakage pattern over a specified timestep variation sequence generates foil vibration, which creates oscillations at the flow-outlet free-surface region on the foil. When the roller rotates at a certain speed, the fluid exerts a potentially increasing force upwards on the foil, and the lift/pressure forces reach a maximum level. This level is sufficient to push the foil upwards, creating a larger gap between the roller and the foil at the nip region. Accordingly, a small degree of leakage may occur. The pressure/lift forces exerted by the fluid subsequently diminish in time and have the effect of resettling the foil back onto the roller. This is a transient (periodic) phenomenon. During the process of foil shifting (up/down), one may equate and balance the respective flow rates between in-flow, coat-outlet flow and nip-outlet flow. The film-layer thickness varies, in a uniform manner, along its length. When there is no-leakage, the required wet film thickness implies a flow rate balance between in-flow and coatoutlet flow. When the foil rises off the roller, a minimal degree of leakage, between $1 \%-3 \%$ of the coat-outlet, is allowed through the nip-gap. This affects the wet film-thickness, reducing its thickness by the same order as that of the leakage, so that, now the coat-outlet flow rate is decreased by the leakage flow rate at the nip. 
In figs.14-15, free-surface profiles are given at $2 \%$ nip-width for the $100_{1}-100_{\mathrm{nl}}$ protocol. Identical free-surface profiles are obtained for other protocols. Wave patterns on the free-surface are apparent. The intensity of such instabilities is demonstrated in fig. 15 on the coat-outlet layer and at the meniscus. These instabilities develop in time and reach a steady-state after long time periods. Such effects onset at the meniscus and propagate towards the coat-outlet region in time, see fig.16. The amplitude of these oscillations enlarge with increasing nip-width. In fig. 15 oscillations are apparent on the free-surface. These would contribute to the final coating finish, rendering an uneven coating layer. The film-width would vary as a consequence, along the complete length of the sheet-foil. Over a leakage period, a slight decrease in the coat-outlet thickness arises to compensate and conserve flow rate. Free-surface profiles at meniscus and coat outlet $_{\text {regions for }}$ various nip-width settings are shown in fig.16. In fig 17, flow is presented in motion blur format at the various times of fig. 16 on the coat-outlet. At steadystate, a layer of uniform width is achieved, see fig. 17a. When the plate is allowed to move up and down in time by adjusting a nip-gap width, film-thickness varies along the strip length. An appropriate criterion is required to constrain the foil vibration, so that the pressure/lift generated remains within acceptable operating levels. We speculate that this may be achieved by selecting suitable leakage/noleakage times.

\subsection{Temporal foil shifting (locally)}

The motivation here is to discern whether foil vibration, local only to the nip, may have the same influence as that detected from global foil movement. Here, we are particularly concerned with lift and the adjustment of free-surface shape. The temporal solution response detected thus far, would indicate that local foil vibration may stimulate similar surface-finish fluctuations. Understanding the fundamental physical reasons behind such fluctuations is our goal.

The foil is shifted vertically upwards, in a local fashion, so that it takes up a slope of inclination within the nip region extending over a fraction of the foil length $(30 \%, 10 \%, 4 \%)$. Nip conditions are taken as of section 5.2. Maximum values of forces are charted below on a foil nip-node, and compared against those of section 5.3.1 with standard settings. We observe from Table 6 and through figs.1718 , that pressure and lift values, increase with decreasing length of the slope (approximating more closely to the standard setting). It is the elevation of peak values that varies between settings. The more local the foil shifting, the more elevated the peak pressures reached.

\begin{tabular}{|c|c|c|c|c|c|}
\hline $\begin{array}{c}\text { Force } * 10^{3} \\
\text { units }\end{array}$ & $\begin{array}{c}\text { Global foil- } \\
\text { shifting }\end{array}$ & $30 \%$ foil_1 & $10 \%$ foil_1 & $4 \%$ foil_1 & $\begin{array}{l}\text { (Standard } \\
\text { nl) }\end{array}$ \\
\hline Pressure & $31.5-57.6$ & $71.5-83$ & $81.0-88$ & $105-110$ & 160 \\
\hline Lift & $408 .-805$ & $33-917.5$ & $1025-1081$ & $1191-1458$ & 63 \\
\hline Drag & $0.475-1.0$ & $1.1-1.2$ & $1.14-1.43$ & $1.27-1.8$ & 0.037 \\
\hline
\end{tabular}

Table 6. Comparison between global and local foil-shifting, at $2 \%$ nip-width, $10_{1}-10_{\text {nl }}$ protocol 
In the comparison of local versus global shifting, for all variables, it is observed that the extent of disturbance from the nip coincides between global and local tests to within $10 \%$ of the foil-length from the nip. This is also true for the standard no-leakage setting (i.e. time independent). Lift and drag decrease proportionally. It is observed that, despite the differences in forces, both global and local settings have the same influence over the coat-outlet free-surface region, see fig. 19.

\section{CONCLUSIONS}

Generally, it is noted that when fluid travels on a coating roller, that moves in the opposing direction to a foil, a pressure build-up will develop against whichever surface is moving with the greater differential speed. Under the present circumstances, it is generally the foil that moves with greater speed. When the pressure build-up reaches a threshold, the foil will be raised away from the roller. This will create a widening of the gap (nip-width) between foil and roller. As a consequence, there will be relief of pressure that will act to bring the foil back down upon the roller. This sequence of events will generate temporal foil vibration. A major observation of the present study emerges. Disturbances on the coat ${ }_{\text {outlet }}$ free-surface may be associated primarily with foil-vibration, either of a global or local nature. These oscillations begin at the meniscus free-surface region and propagate towards the coat ${ }_{\text {outlet }}$ region. Vibration in the free-surface profiles has been demonstrated at different time-step protocols, under various L\% foil shifting, and in both leakage and no-leakage states. It is observed that the disturbance ratio factor on the coat ${ }_{\text {outlet }}$ free-surface is around $2 \%$ for all leakage settings, once a settled state has developed. 


\section{REFERENCES}

[1] P. Townsend and M.F. Webster, An Algorithm for the Three-Dimensional Transient Simulation of non-Newtonian Fluid Flows, Proc. NUMETA 87, Martinus Nijhoff, Publishers, Dordrecht, 2:T12/1, 1987.

[2] D.M. Hawken, H.R. Tamaddon-Jahromi, P. Townsend and M.F. Webster, $A$ Taylor-Galerkin Based Algorithm for Viscous Incompressible Flow, Int. J. Num. Meth. Fluids, 10, 327-351 (1990).

[3] E.O.A. Carew, P. Townsend, M.F. Webster, A Taylor-Petrov-Galerkin Algorithm for Viscoelastic Flow, J. non-Newtonian Fluid Mech., 50, 253-287 (1993).

[4] O. Cohu and A. Magnin, Forward Roll Coating of Newtonian Fluids with Deformable Rolls: An Experimental Investigation; Chem. Eng. Sci., vol. 52, no.8, 1339-1347 (1997).

[5] M.S. Carvalho and L.E. Scriven, Multiple States of a Viscous Free-Surface Flow: Transition from Pre-metered to a Metering Flow; Int. J. Num. Meth. Fluids, vol. 24, 813-831 (1997).

[6] D. Marcio. S. Carvalho and L. E. Scriven, Deformable Roll Coating Flows: Steady State and Linear Perturbation Analysis; J. Fluid Mech., vol. 339, 143172 (1997).

[7] E. Fourcade, F. Bertrand, O. Régalt, P.A. Tanguy, Finite Element Analysis of Fluid-Solid Interaction in the Metering Nip of a Metering Size Press; Comp. Meth. Appl. Mech. Eng., vol. 174, 235-245 (1999).

[8] K.S.A. Chen and L.E. Scriven, Roll Coating, Forward and Reverse: Effect of Feed Condition; AIChE Spring Nat. Meet., New Orleans, LA 1988

[9] D.F. Benjamin, Roll Coating Flows and Multiple Flow Systems; PhD. Thesis, University of Minnesota, Minneapolis, MN, University Microfilms Int., Ann. Arbor, MI 1994.

[10] R. Keunings, An Algorithm for the Simulation of Transient Visco-Elastic Flows with Free-Surfaces, J. Comp. Phys., 62, 199-220 (1986)

[11] R. Sizaire and V. Legat, Finite Element Simulation of a Filament Stretching Extensional Rheometer, J. non-Newtonian Fluid Mech., vol.71, 89-107 (1997).

[12] R.I. Tanner, R.E. Nickell, R.W. Bilger, Finite Element Method for the Solution of Some Imcompressible non-Newtonian Fluid Mechanics Problems with Free Surfaces, Comp. Meth. App. Mech. Eng., vol. 6, 155-174 (1975).

[13] W.J. Silliman and L.E. Scriven, Separating flow near a static contact line: slip at $a$ wall and shape of a free surface, J. Comp. Phys., vol. 34, 287-313 (1980).

[14] H. Saito and L.E. Scriven, Study of Coating Flow by the Finite Element Method, J. Comp. Phys., 42, 53-76 (1981).

[15] B. Ramaswamy, Numerical Simulation of Unsteady Viscous Free-Surface Flow, J. Comp. Phys., vol. 90, 396-430 (1990).

[16] C.W. Hirt, A.A. Amsden, and J.L. Cook, An Arbitrary Lagrangian-Eulerian Computing Method for All Flow Speeds, J. Comp. Phys., 14, 227-253 (1974). 
[17] T. Sato and S.M. Richardson, Numerical Simulation Method for Viscoelastic Flows with Free Surface-Fringe Element Generation Method, Int. J. Num. Meths. Fluids, vol. 19, 555-574 (1994).

[18] M.S.Chandio and M.F. Webster, Numerical simulation of reverse rollercoating of aluminium alloy sheets with free-surface, ECCOMAS Computational Fluid Dynamics Conference, Swansea, Wales, UK, September 2001.

[19] O. Regalt, R. Labrie, and P.A. Tanguy, Free-surface Model for Dip Coating Process, J. Comp. Phys., 109, 238-246 (1993).

[20] C. Cuvelier, A. Segal, A. A. Van Steenhoven, Finite Element Methods and Navier-Stokes Equations, D. Reidel Publishing Company, 1986. 


\section{FIGURE LEGEND}

1. Schematic flow diagram

2. Finite element mesh sections

3. Nip mesh and velocity conditions

4. Schematic diagram of plug and Couette flow velocity profiles at nip

5. Flow pattern with various nip velocity/pressure conditions

6. Distributional pressure, lift along foil and drag on roller

7. Temporal leakage adjustment: settled distributional pressure and lift along foil and drag on roller, $10_{1}-10_{\mathrm{nl}}$ protocol

8. Temporal leakage adjustment, pressure at nip node $\mathrm{N}_{1}$

9. Global foil shifting, steady state: distributional pressure and lift along foil and drag on roller

10. Global foil shifting, temporal variations, settled distributional pressure, lift along foil and drag on roller at $L \%$ nip-width of coat ${ }_{\text {outlet, }} 10^{1}-10_{\mathrm{nl}}$ protocol

11. Global foil shifting, temporal variations, settled distributional pressure, lift along foil and drag on roller at $L \%$ nip-width of coat ${ }_{\text {outlet }}, 100_{1}-100_{\mathrm{nl}}$ protocol.

12. Global foil shifting; pressure line plots at nip node $\mathrm{N}_{1}, \mathrm{~L} \%$ nip width

13. Global foil shifting; pressure line plots at nip node $\mathrm{N}_{1}$, leakage state $P_{\min }(\mathrm{t})$

14. Global foil shifting; free-surface profile- full view

15. Global foil shifting; free-surface profiles, meniscus and coat-outlet; $2 \%$ nip-width, $100_{1}-100_{\text {nl }}$ protocol, zoomed view at different time-steps

16. Global foil shifting; free-surface profiles, meniscus and coat-outlet; comparison at $2 \%, 3 \%$ and $5 \%$ nip-width, $100_{1}-100_{\mathrm{nl}}$ protocol, zoomed view

17. Global foil shifting; coat-outlet free-surface profiles, motion blur format, different times.

18. Comparison of settled distributional pressure and lift; global foil shifting verses local foil shifting (slope over $30 \%$ of foil length)

19. Comparison of settled distributional pressure and lift; global foil shifting verses local foil shifting (slope over $4 \%$ of foil length)

\section{TABLE LEGEND}

1. Nip conditions; velocity

2. Nip conditions; pressure, lift and drag

3. Temporal leakage adjustment; pressure, lift and drag

4. Global foil shifting to steady state; pressure, lift and drag

5. Foil shifting (globally); temporal variations; pressure, lift and drag

6. Comparison between global and local foil-shifting, at $2 \%$ nip-width, $10_{1}-10_{\text {nl }}$ protocol 

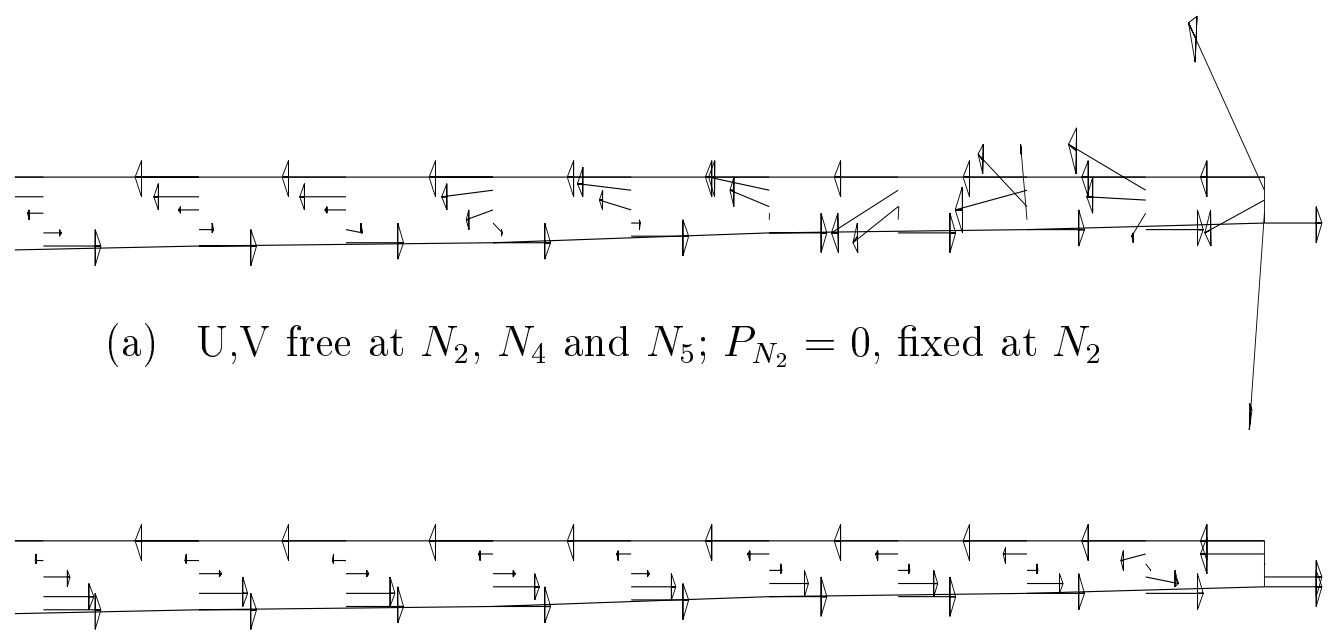

(b) $U_{N 4}=U_{N 1} ; U_{N 5}=U_{N 3} ; U_{N 2}=0 ; P_{N 2}$ free

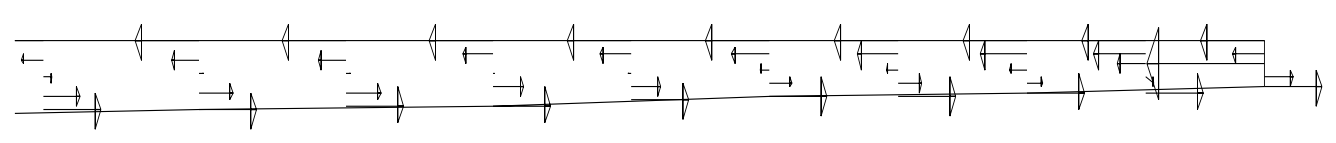

(c) $U_{N 4}=U_{\text {foil }} \frac{1}{h_{0}}(y) ; U_{N 5}=U_{\text {roller }} \frac{1}{h_{0}}(-y) ; U_{N 2}$ free

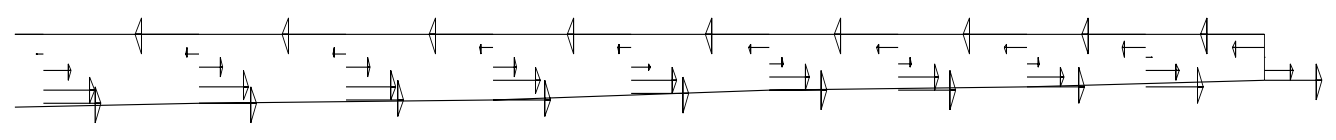

(d) $U_{N 4}=U_{\text {foil }} \frac{1}{h_{0}}(y) ; U_{N 5}=U_{\text {roller }} \frac{1}{h_{0}}(-y) ; U_{N 2}=0$ fixed

Figure 5: Flow patterns with various nip velocity/pressure conditions 

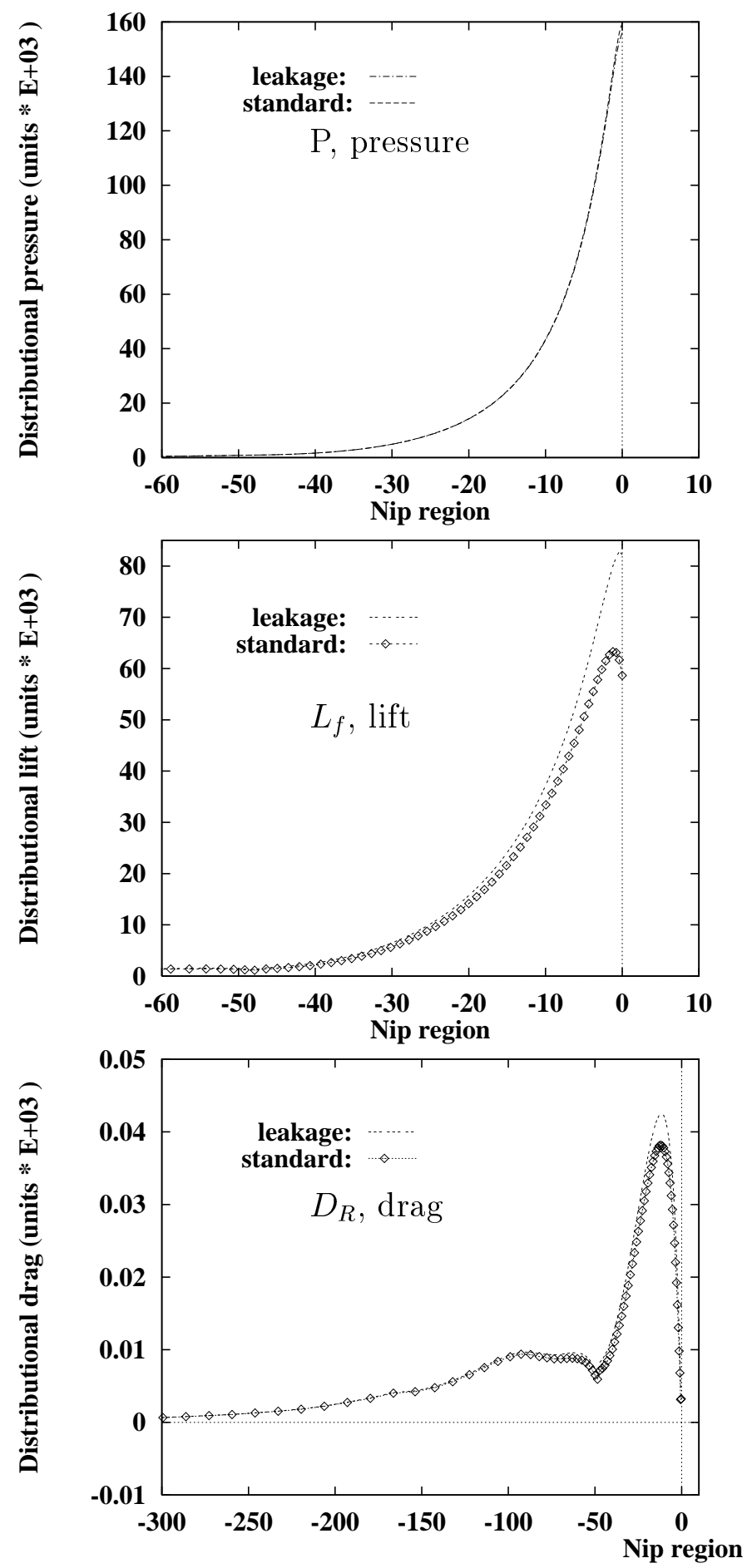

Figure 6: Distributional pressure, lift $L_{f}$ along foil and drag $D_{R}$ on roller. 

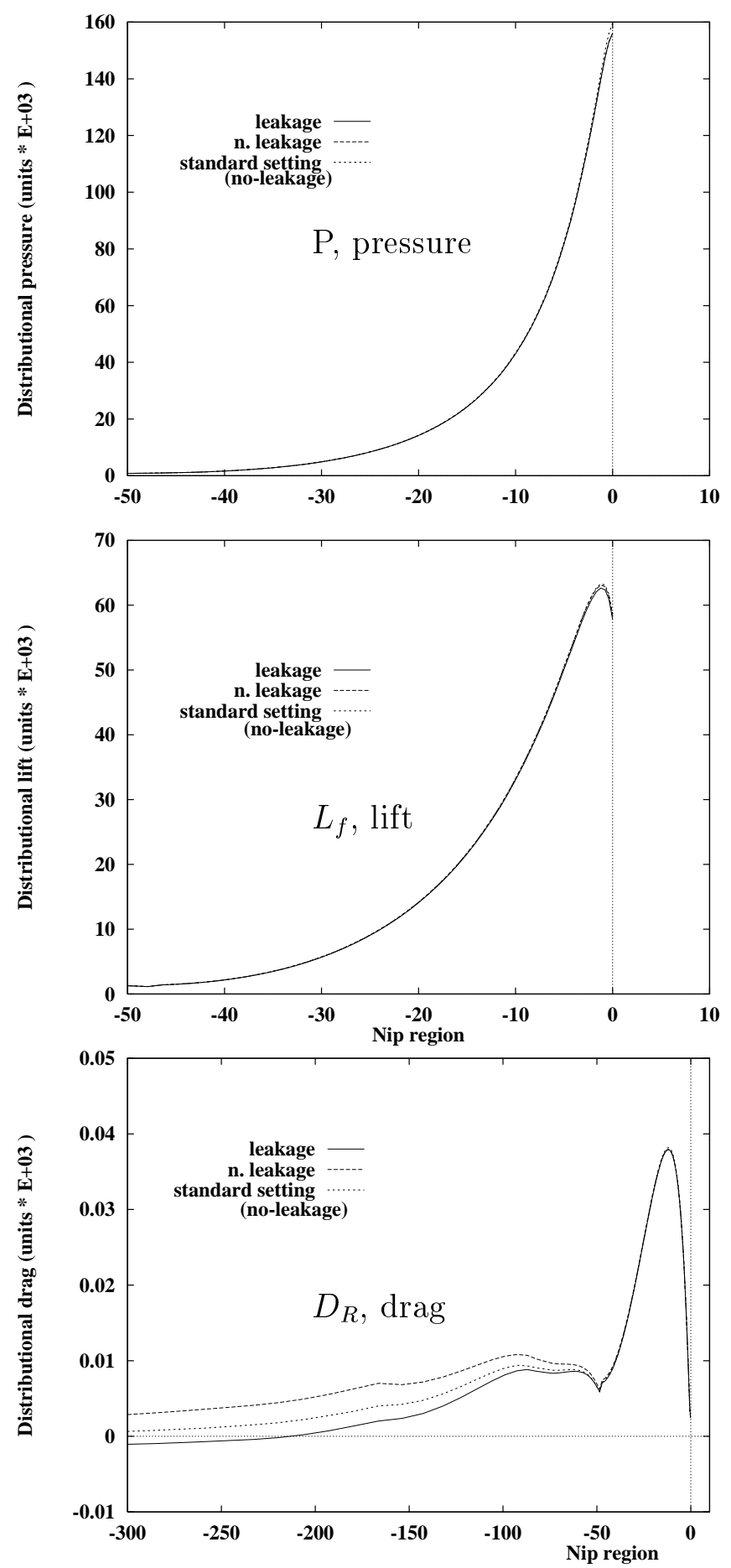

Figure 7: Temporal leakage adjustment: settled distributional pressure and lift along foil and drag on roller , $10_{l}-10_{n l}$, true for all protocols 


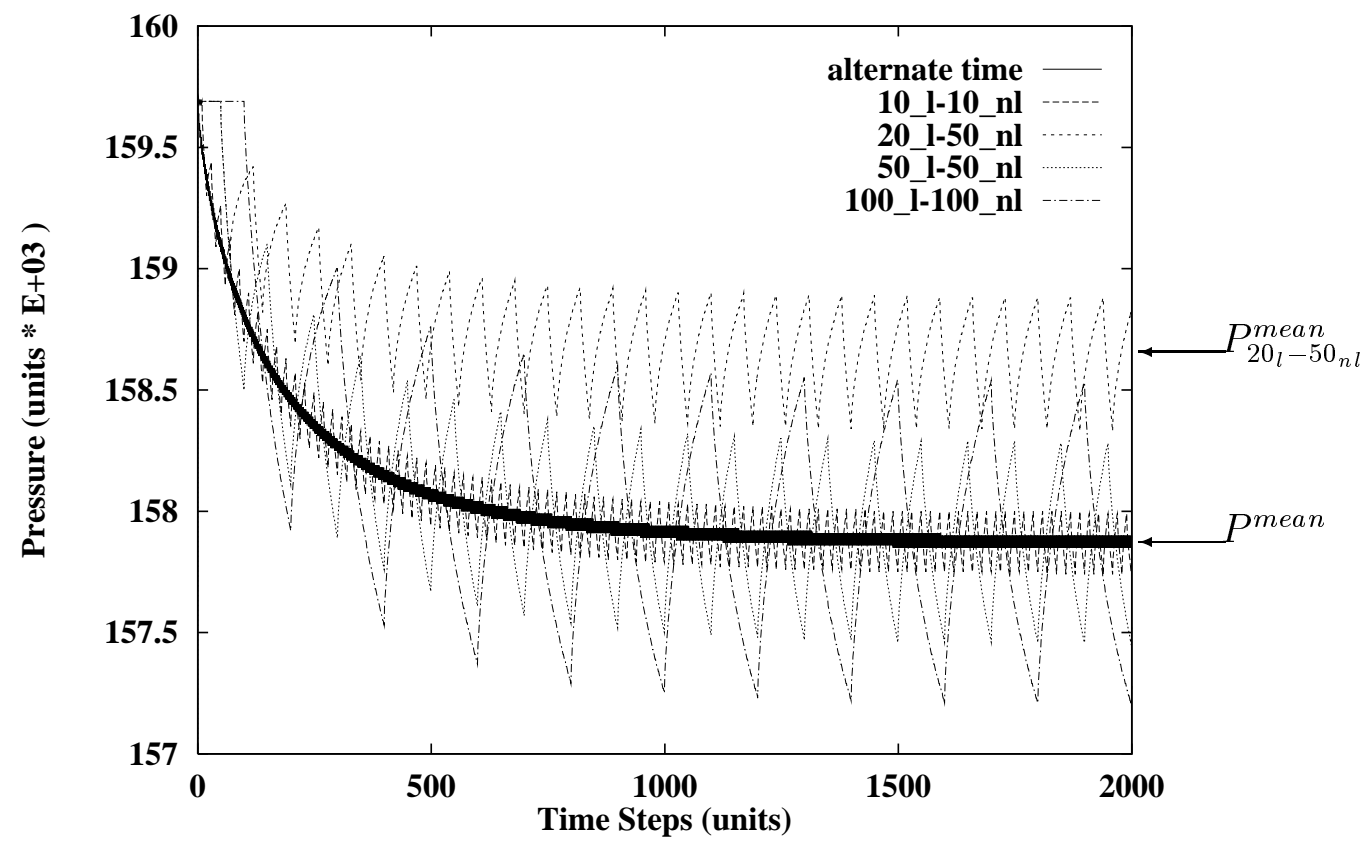

Figure 8: Temporal leakage adjustment, pressure at nip node $N_{1}$. 

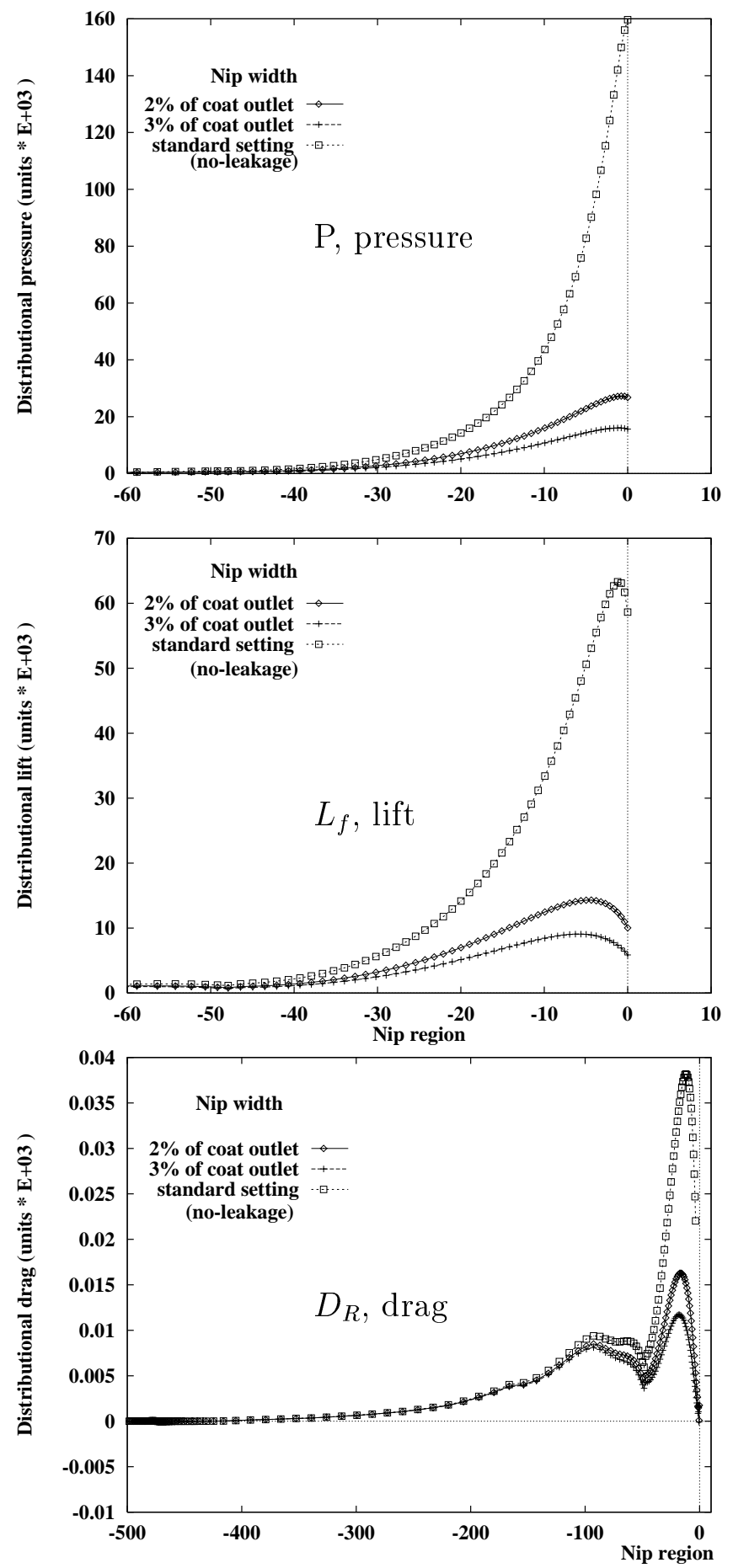

Figure 9: Global foil shifting, steady state: distributional pressure and lift, $L_{f}$, along foil, drag, $D_{R}$, on roller. 

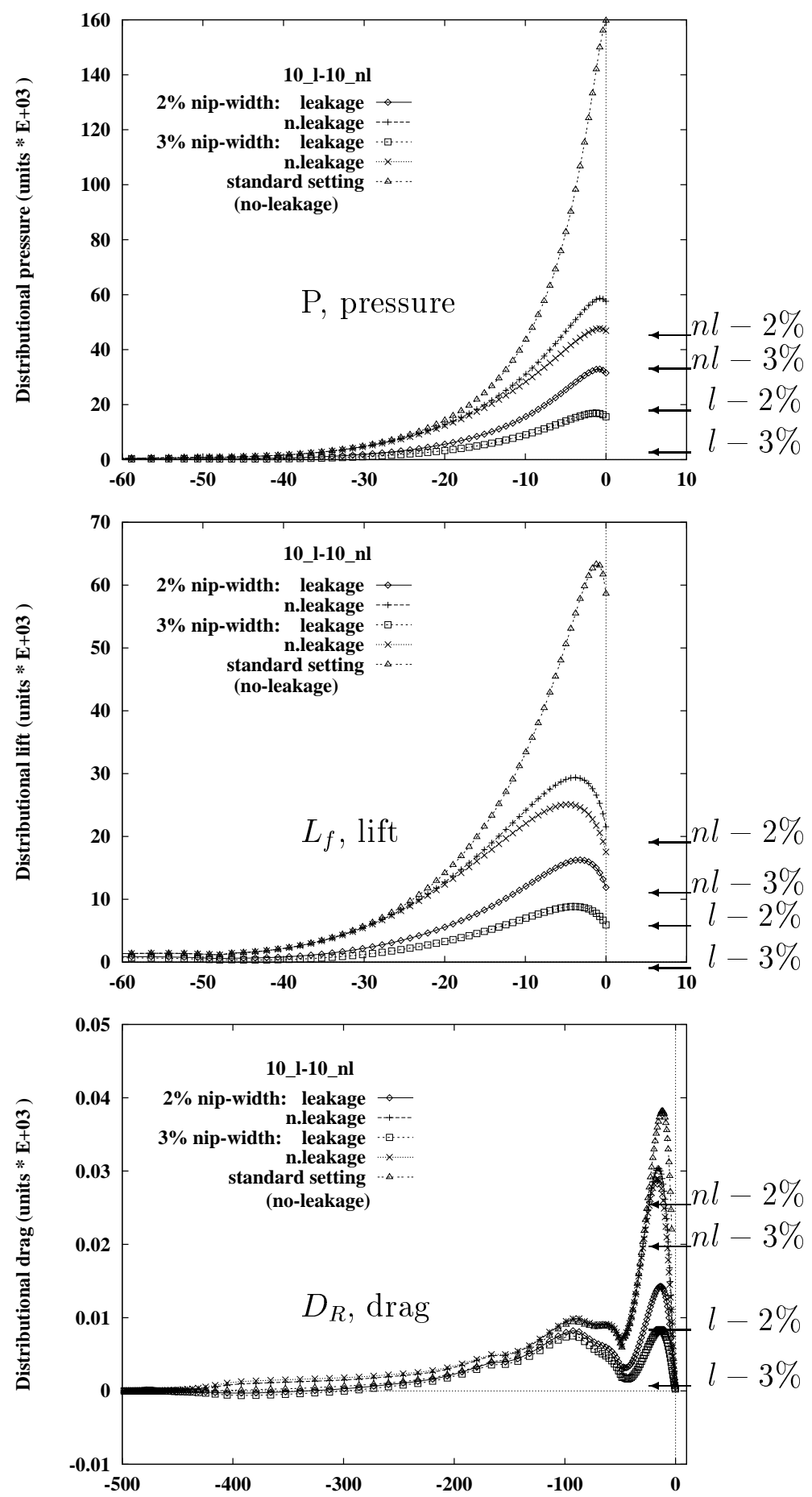

Figure 10: Global foil shifting, temporal variations, settled distributional pressure, lift along foil and drag on roller at $L \%$ nip-width of coat outlet $_{\text {, }}$ $10_{l}-10_{n l}$ protocol 

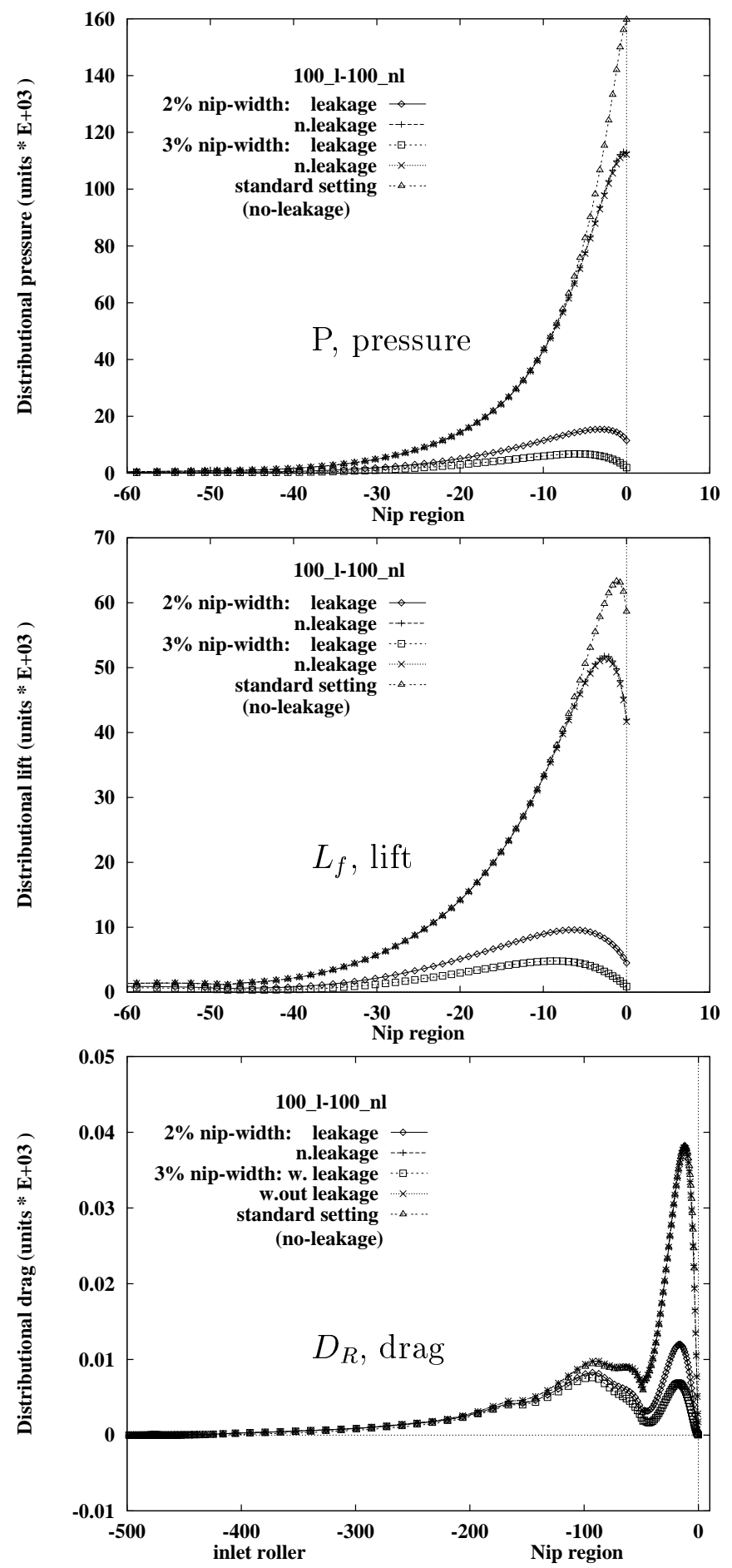

Figure 11: Global foil shifting, temporal variations, settled distributional pressure, lift along foil and drag on roller at $L \%$ nip-width of coat outlet $_{\text {, }}$ $100_{l}-100_{n l}$ protocol 

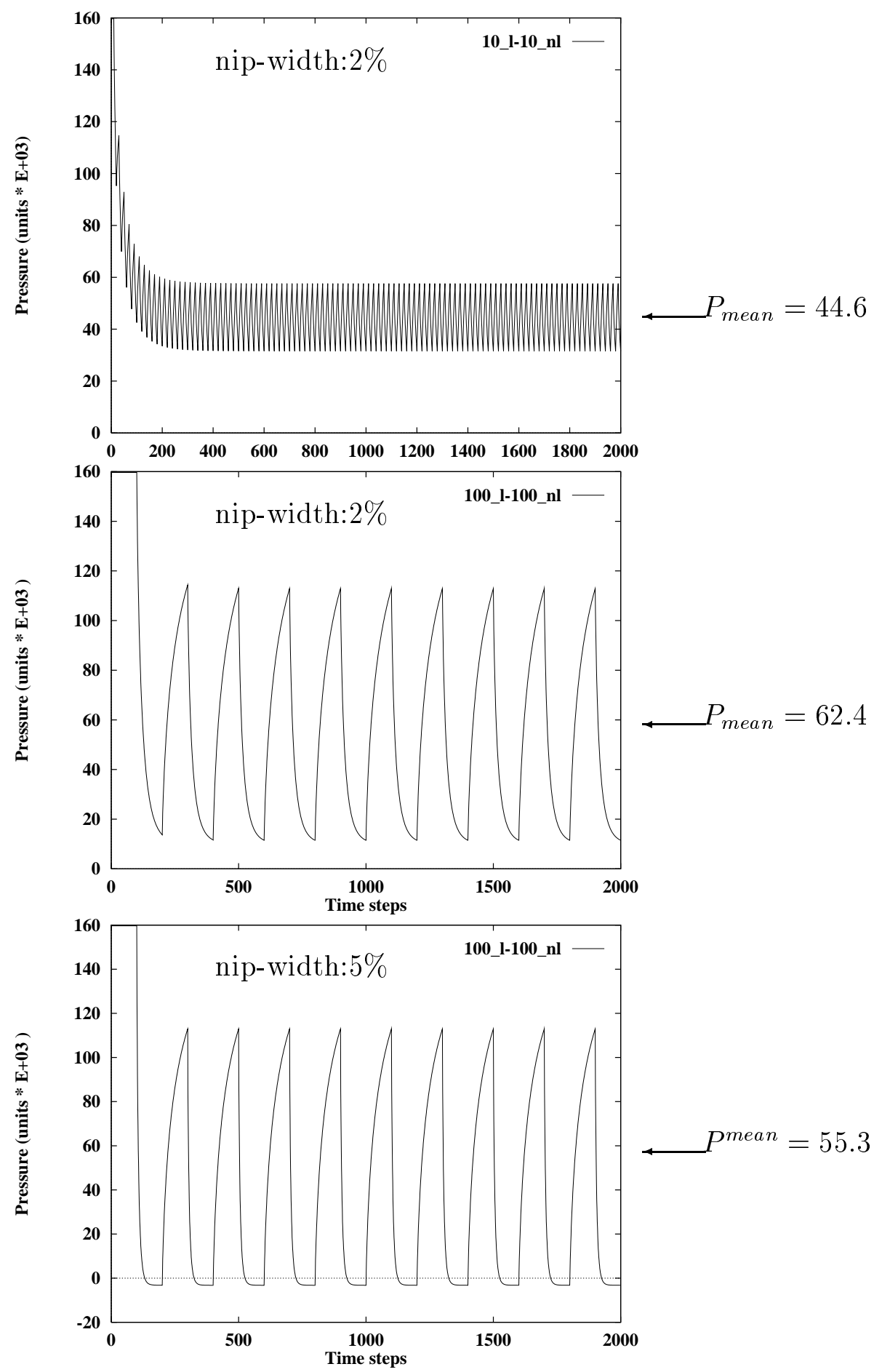

Figure 12: Global foil shifting; pressure line plots at nip node $N_{1}, \mathrm{~L} \%$ nip width 

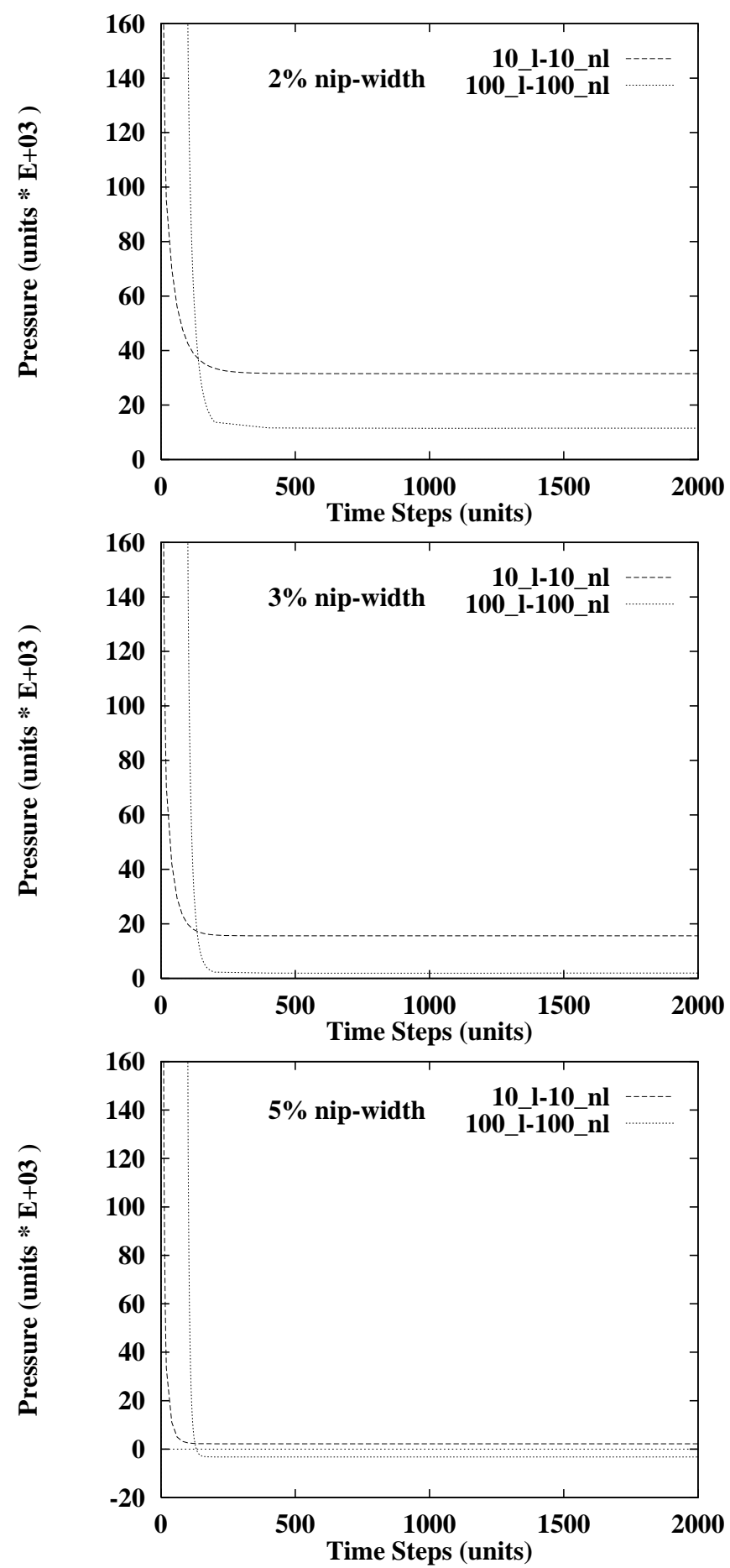

Figure 13: Global foil shifting; pressure line plots at nip node $N_{1}$, leakage state $P_{\min }(\mathrm{t})$ 


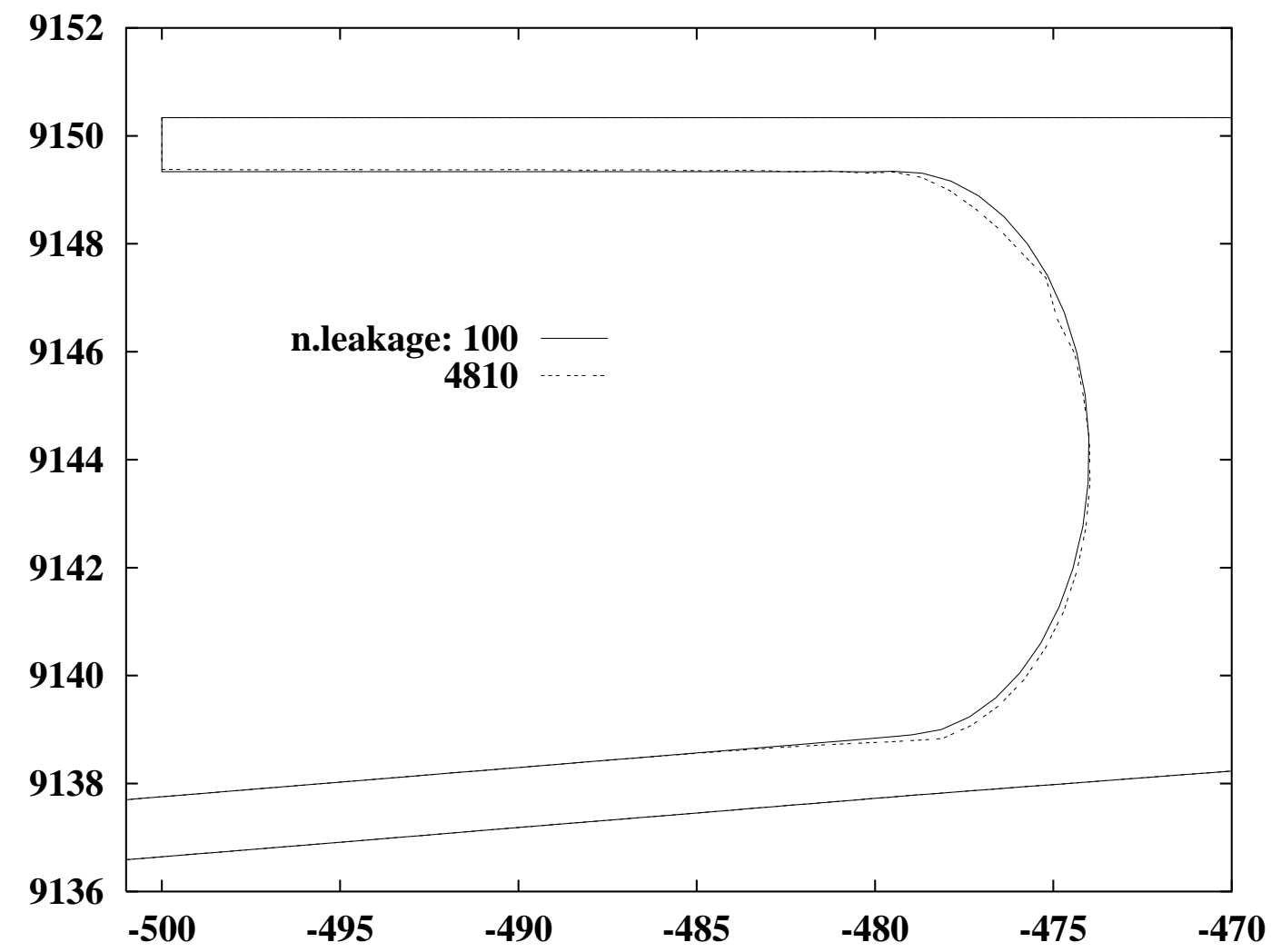

Figure 14: Global foil shifting; free-surface profile - full view 


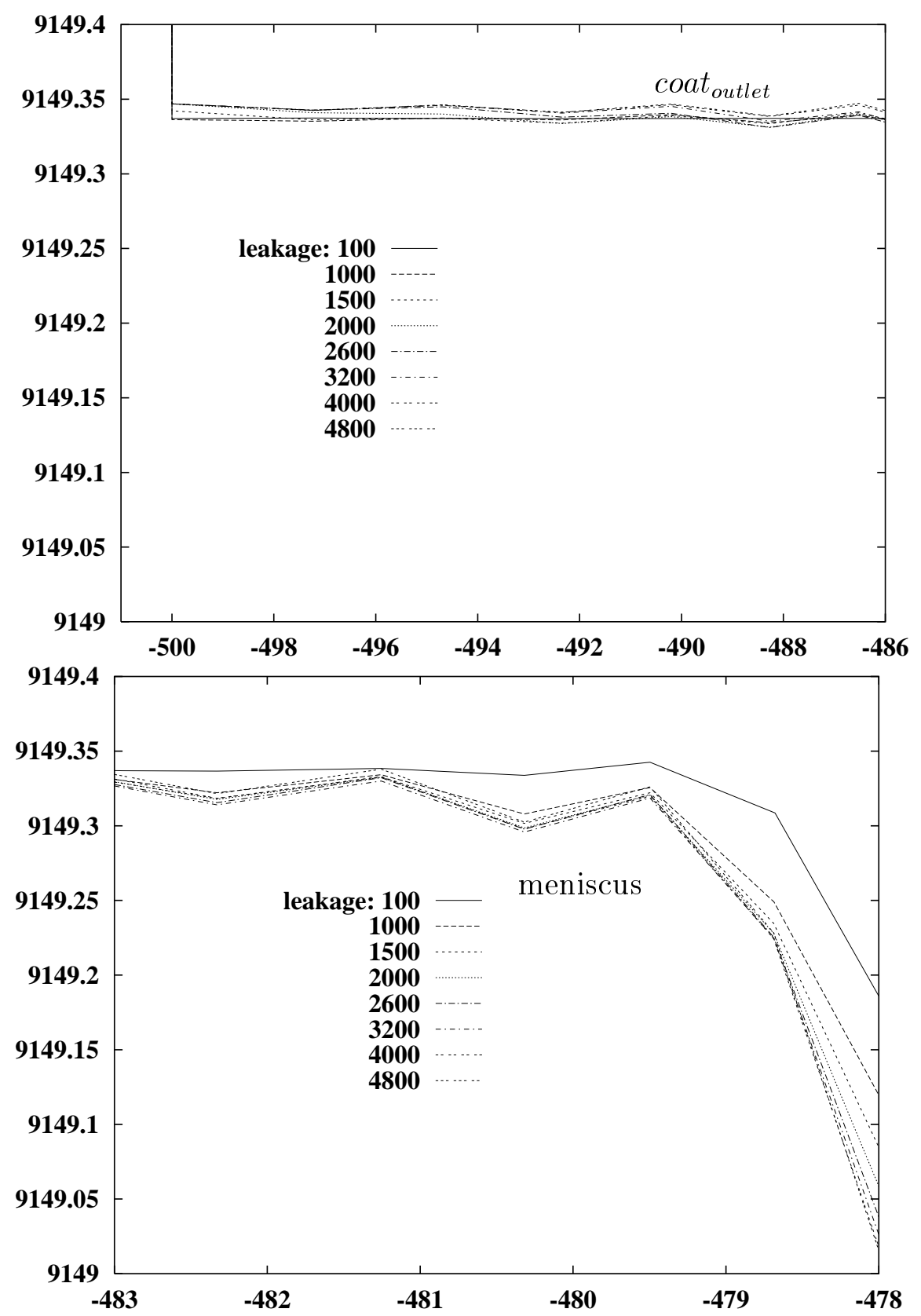

Figure 15: Global foil shifting; free-surface profiles- meniscus and coat-outlet; $2 \%$ nip-width, $100_{l}-100_{n l}$, zoomed view at different time-steps. 

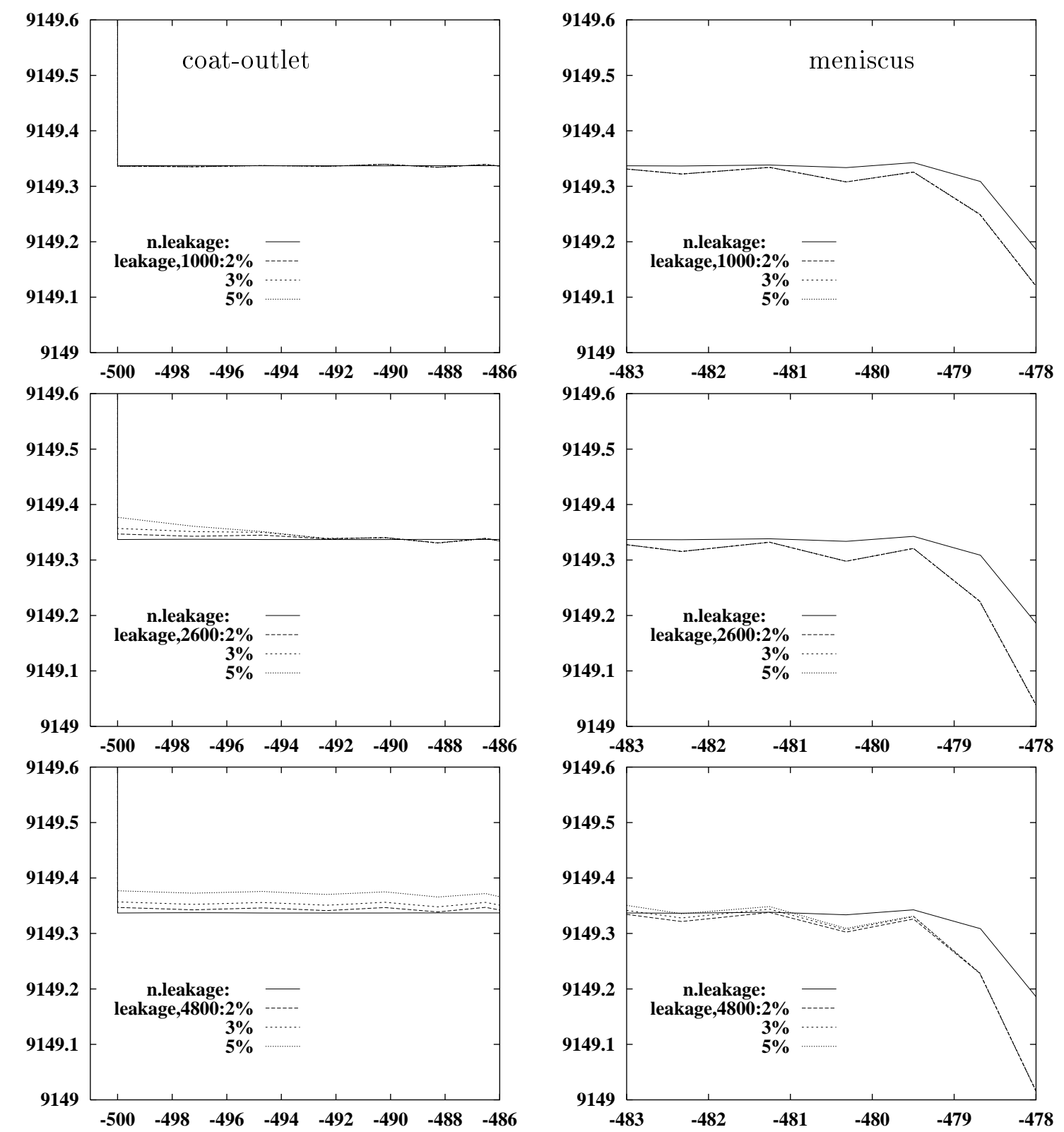

Figure 16: Global foil shifting; free-surface profiles- meniscus and coat-outlet; comparison at $2 \%, 3 \%$ and $5 \%$ nip-width, $100_{l}-100_{n l}$, zoomed view. 
(a)

(b)

(c)

(d)

Figure 17. Global foil shifting; coat-outlet free-surface profiles, motion blur format, different times. 

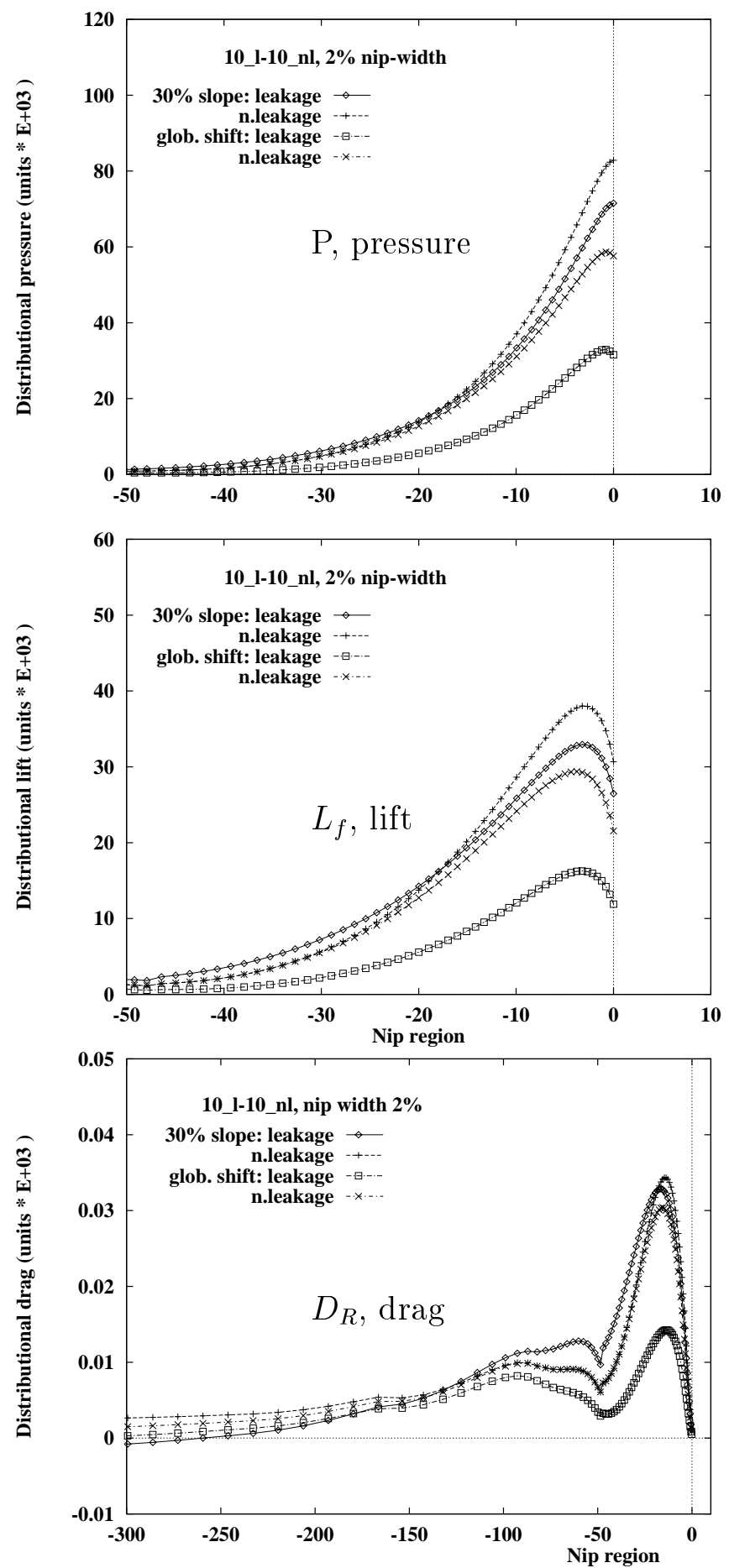

Figure 18: Comparison of settled distributional pressure and lift: global foil shifting verses local foil shifting (slope over $30 \%$ of foil length) 

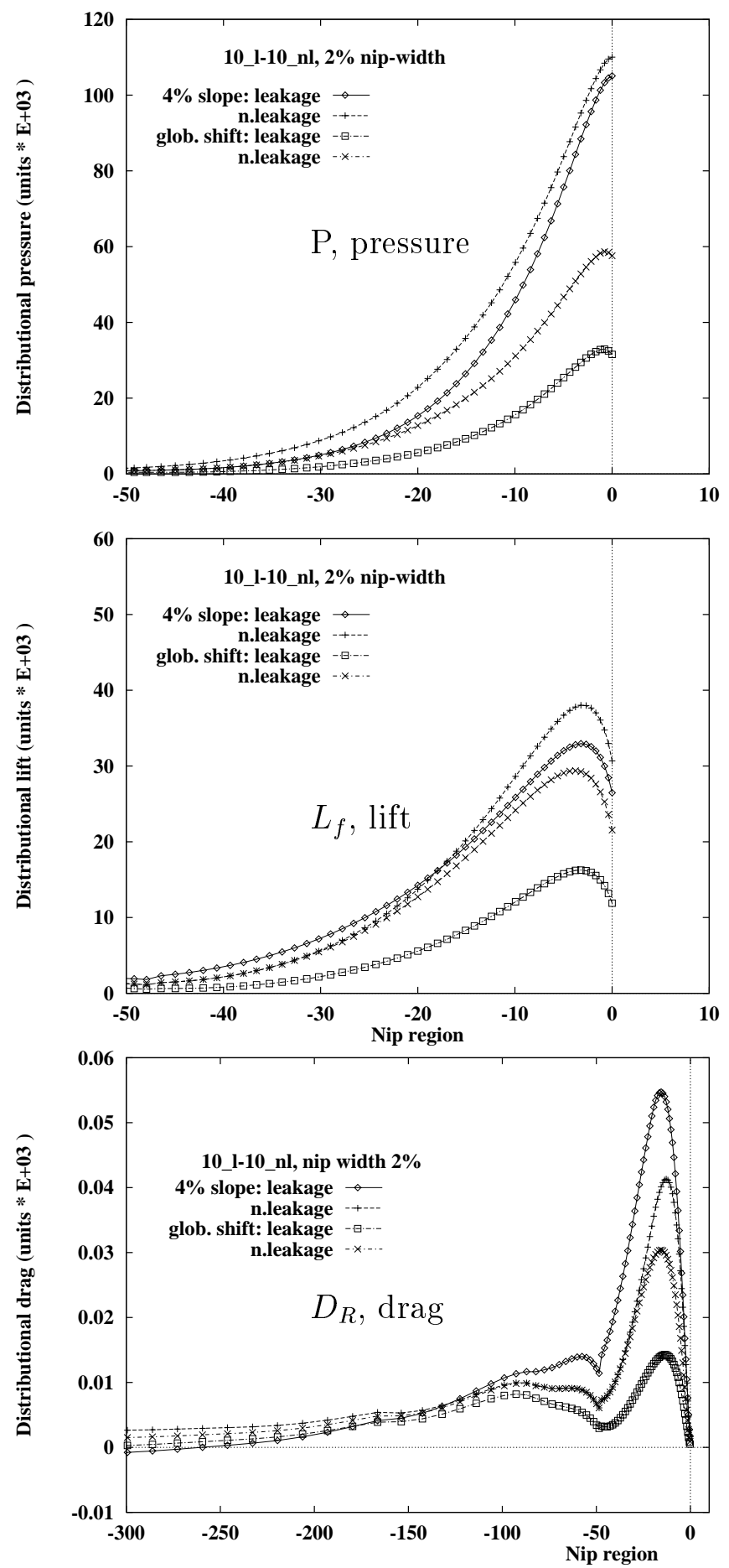

Figure 19: Comparison of settled distributional pressure and lift: global foil shifting verses local foil shifting (slope over $4 \%$ of foil length) 